\title{
RÉVISION DE QUELQUES HYPOTHÈSES SUR LE CREUSEMENT KARSTIQUE*
}

"Ne recevoir jamais aucune chose pour vraie que je ne la connusse évidemment être telle» DESCARTES - 1637, Discours de la méthode

\author{
Jacques CHOPPY \\ Ingenieur - Geophysicien \\ 182, rue Vaugirard F- 75015 PARIS (France)
}

\section{RÉSUMÉ}

Le plus souvent parce que nous connaissons beaucoup mieux les phénomènes karstiques et l'évolution de l'environnement du karst, diverses hypothèses relatives au creusement karstique apparaissent fragiles.

Quelques-unes de ces hypothèses, relatives à des processus intervenant dans la création des formes du karst, dépassent leurs limites d'action. D'autres imaginent des fonctionnements hydrauliques, des interventions de facteurs géologiques ou géographiques susceptibles d'être contestés. Les hypothèses concernant l'évolution du karst, comme les classifications de ceuxci, souffrent de ne pas avoir été précédées d'une approche analytique.

Certaines de ces hypothèses occupent une place importante dans la vision actuelle de beaucoup de spéléologues et karstologues.

Mots clef: phénomènes karstiques, hypothèses, histoire.

\section{ABSTRACT (REVISION OF A FEW HYPOTHESES ON SPELEOGENESIS)}

Quite often, as we know better karst phenomena and the evolution of the karst environment, several hypotheses on speleogenesis $\mathrm{s}$ appeared to be flimsy.

Some of these hypotheses, concerning processes playing a part in the creation of karst forms, exceed the limits of their field. Others suggest hydraulic mechanisms, and interventions of geological or geographical factors likely to be questioned. Hypotheses relating to the evolution of karst, as well as the classification of karst types, suffer from the lack of an analytical approach.

However, some of these hypotheses still have an important place in the current vision of speleologists and karstologists.

Keywords: speleogenesis, theories, history.

\footnotetext{
* Both the referees felt the paper should not be accepted due to its content which cannot fit with well with the IJS even if extensively changed, moreover the author choose not to make many of the changes that were suggested. However, the Editors have decided to print the paper because they consider it is thought-provoking and may stimulate discussion on some basic speleogenetic concepts, which are currently accepted in the scientific community.
} 


\section{Introduction}

Dans la préface de sa thèse, Philippe AUDRA [1994] me qualifie gentiment de " pourfendeur du dogmatisme ». Je ne récuse pas cette appellation, même si mes prétentions sont plus modestes. Certes, j'ai progressivement cessé de croire à certaines hypothèses couramment admises en matière de creusement karstique, ou parfois à l'usage qu'on en fait.

Dans le domaine des sciences de la terre, l'explication des formes demeure toujours pour une part hypothétique. C'est vrai en particulier pour le karst, est-il nécessaire de le dire ? Si certaines hypothèses peuvent apparaître fragiles, dans leur principe ou dans certaines applications, elles résultent de travaux souvent novateurs, dont certains ont entraîné de nombreux développements. Mais, à l'époque, la connaissance des phénomènes karstiques et de leur environnement étaient infimes par rapport à ce dont nous disposons aujourd'hui.

Or plusieurs de ces hypothèses sont devenues des dogmes, au point que les expressions génétiques qu'elles ont créées sont quotidiennement employées de manière descriptive ; ce qui anticipe évidemment sur toute interprétation. Si le dogme s'effondre, il entraîne avec lui les interprétations qui en furent déduites ! A-t'on le droit de dire que tout le monde n'est pas d'accord?

Afin de rester dans le cadre d'un article. je me borne à dire pourquoi.

Et il n'est pas rare qu'on voie resurgir dans une publication récente d'autres hypothèses qui semblaient heureusement oubliées, si même elles ne redeviennent pas à la mode. Sans prétendre à l'exhaustivité, doit-on se limiter dans l'inventaire de celles qui semblent mal fondées?

Dans le passé, l'étrangeté de beaucoup de phénomènes karstiques a frappé de nombreux savants, qui en ont cherché des explications : celles-ci, fondées sur des connaissances très locales, furent souvent gratuites, mais parfois prémonitoires ; elles n'ont pratiquement jamais eu d'audience générale. T.R. Shaw [1979] s'étant fait l'historien de ces recherches, il ne semble pas utile de remonter ici avant la fin du $19^{\mathrm{e}}$ siècle, époque où apparaît une volonté exploratoire organisée, avec diffusion des résultats et des hypothèses aux autres explorateurs.

\section{Hypothèses relatives à des processus}

L'importance relative des processus physico-chimiques, hydrauliques, micro-climatiques, mécaniques, biologiques fut l'objet d'énergiques prises de position, ou de débats passionnés (concernant les rôles respectifs de la corrosion ou de l'érosion ; de la dissolution ou de l'effondrement ; etc.). Or, pour la plupart, ces processus sont présents dans tous les karsts ; mais ils ne sont pas tous mobilisés.

Cependant tout processus, même bien réel, a ses limites. 


\section{Corrosion en fonction de la température}

Puisque le taux de dissolution du $\mathrm{CO}_{2}$ dans l'eau est supérieur lorsque celle-ci est froide, J. Corbel [1957] suppose que la corrosion du calcaire est augmentée sous un climat froid. Depuis, plusieurs auteurs ont montré que le rôle de la température sur la dissolution ne pouvait être pris en compte isolément : la dynamique des réactions, puis des facteurs divers, dont le volume des précipitations, sont souvent plus importants [M. Julian 1992].

\section{Limite de karstification en profondeur}

On sait qu'à l'équilibre, de l'eau se trouvant sous un gaz en dissout une quantité d'autant plus grande que la pression est élevée et la température faible (loi de HENRY). Pour une eau descendant en profondeur, la pression et la température augmentent.

L'équilibre de la loi de HENRY, assez rapidement atteint après une variation de température, l'est beaucoup moins après une variation de pression. A. Vandenberghe [1960] suppose alors que la solubilité du $\mathrm{CO}_{2}$ diminuant vers la profondeur « sous l'action de la température,... l'influence de la pression n'intervient que faiblement pour corriger ce phénomène »; ceci jusqu'à « une limite de profondeur au delà de laquelle » il prévoit « une précipitation des carbonates ».

Cette précipitation n'est pas observable dans les carottes de forage [H. Schoeller 1965]. Quant au raisonnement de A. Vandenberghe [1960], il invoque des processus liés à la loi de HENRY dans des conditions où celle-ci n'est pas applicable, puisqu'en profondeur le liquide ne se trouve pas en regard du gaz. À grande profondeur dans des forages pétroliers, on constate au contraire la présence d'eaux très carbonatées [J Corbel 1957]. Lors de la remontée de ces eaux par le trou de forage, on ne signale d'ordinaire ni dégazage de $\mathrm{CO}_{2}$, ni dépôt de calcaire ; ces eaux ne sont donc pas sursaturées par rapport aux conditions de surface : elles n'ont généralement pas eu, en profondeur, l'occasion de s'enrichir en $\mathrm{CO}_{2}$. Reprenant le problème sur un plan plus large, H. Schoeller [1965] conclut à l'inexistence d'une limite de karstification dans les cas habituels.

\section{Corrosion par mélange des eaux [A Bögli 1964]}

Tout mélange de deux eaux, acidifiées par la dissolution de $\mathrm{CO}_{2}$ et saturées en $\mathrm{CaCO}_{3}$, mais de chimismes différents, se trouve dans la zone d'agressivité par rapport à la courbe de saturation. Tout en confirmant que, globalement, le phénomène existe, Y. Quinif [1981] note qu'en présence d'air, un rééquilibrage entre les taux de $\mathrm{CO}_{2}$ de l'air et de l'eau peut s'opposer à la corrosion supplémentaire par mélange des eaux.

Dans le cas habituel d'un massif karstique, où les eaux des précipitations suivent des 
chemins parallèles, de sorte que leur chimisme est le même au moment du mélange, celui-ci est inopérant. Et quand les eaux mélangées sont de chimismes différents, la corrosion supplémentaire du calcaire ne peut guère excéder $10 \%$. Les formes résultantes doivent alors être de taille modeste par rapport au conduit dans lequel elles se trouvent, et ne peuvent avoir un aspect spécifique que si elles se situent au lieu même où se produit le mélange.

\section{Creusement superficiel sous couverture}

Des formes en relief, mais aussi d'aplanissement, ont été attribuées à un creusement sous couverture :

- Le karst à buttes se développerait « dans les dépressions où s'accumulent des sédiments »[A. Bonte 1958]. Si le creusement sous couverture y participe vraisemblablement, l'élaboration d'un karst à buttes résulterait plutôt, selon A. Mangin et $\mathrm{M}$. Bakalowicz [1990], d'une surrection lente et continue permettant à l'érosion « normale » de garder la prépondérance par rapport au façonnement karstique. Cette hypothèse fut également proposée par R. Maire et S. Pomel [1995] à propos du karst de Wufeng (Hubei, Chine), mais sans que soit expliqué comment le surcreusement des vallées à la suite d'une surrection, permettant le décapage d'une couverture, peut aboutir à un relief en buttes.

Dans la représentation de H. Lehmann [1953 - figure 1], une évolution du karst à buttes vers un karst à tours résulte du démarrage d'une «Lösungsunterschneidung »

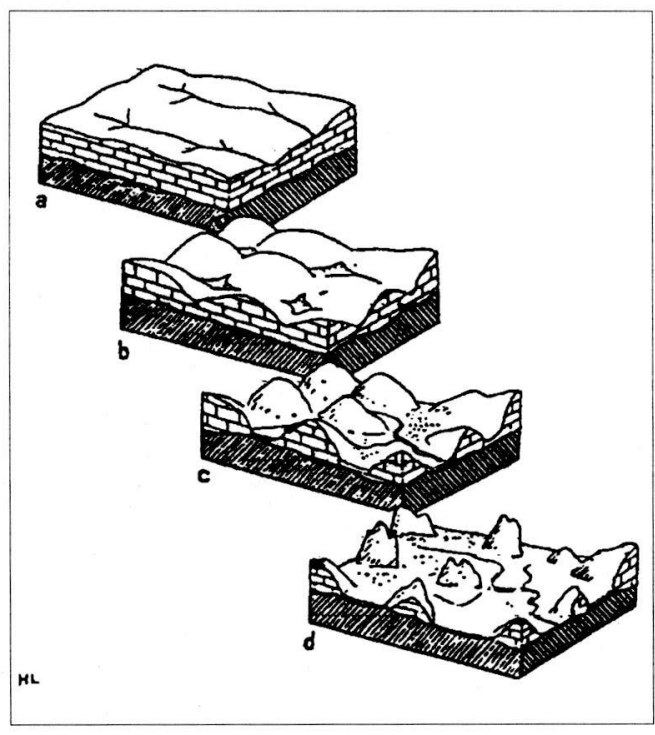
(= corrosion ayant un effet de sapement) quand le creusement atteint le niveau d'un soubassement sédimentaire. Cette hypothèse, implicitement reprise par d'autres auteurs, correspond sans doute à des cas particuliers. Mais si la localisation des karsts à tours montre que leur base est ou fut inondable, provoquant une corrosion latérale, c'est plus souvent dans des conditions particulières, comme la proximité immédiate du niveau de la mer.

L'évolution du karst à buttes vers un karst à tours ne serait pas un phénomène général.

Fig. 1- Schéma de l'évolution des buttes sous les tropiques (d'après LEHMANN, 1953) (The evolution of cone karst in the tropics) 
- J. Nicod [1967] attribue les reliefs ruiniformes à un creusement sous couverture ; celui-ci peut contribuer à leur formation, mais n'explique pas leur localisation sur des versants de vallées ou de dépressions, en particulier des formes les plus volumineuses.

- La « surface d'aplanissement karstique » résulterait d'une corrosion sous une couverture perméable [J. Cvijic 1909]. Au contraire, pour D. Aubert [1975], un « aplanissement karstique » résulterait du recul des têtes de bancs affleurant en surface, émoussant ainsi les sommets d'anticlinaux. Et, dans le Jura français, selon G. Chabot [1927], la morphologie des surfaces aplanies relève d'une « érosion normale » et non d'une action karstique.

\section{Creusement sous pression}

P. Chevalier [1944] suppose que le creusement des conduits débute par une «érosion sous pression, en conduite forcée » (sans indiquer les conditions expliquant cette pression), utilisant les joints de stratification et les fractures mais indépendante du pendage comme de la pesanteur, et aboutissant à une forme en tube. Les expressions génétiques «érosion sous pression 》, « érosion en conduite forcée » désignent donc un processus hypothétique. P. Chevalier [1944] ne s'est pas beaucoup expliqué mais, en tant qu'ingénieur et compte tenu de l'époque, celle des grandes réalisations hydroélectriques, l'expression « conduite forcée » est une référence précise à des conduites dans lesquelles les pressions, les vitesses et les débits sont habituellement importants.

Sous le niveau de base, le « creusement sous pression » ne se distingue pas du creusement « phréatique » examiné ci-dessous. Et, au dessus, on ne voit pas comment une pression importante pourrait se maintenir, sinon de manière épisodique, car des fuites vers l'extérieur devraient se produire.

Un creusement par pression hydrostatique est parfois invoqué. Pour le processus, soit il n'est pas expliqué [E.A. Martel 1921], et du reste difficile à imaginer, soit il s'agit d'une différence de pression à l'intérieur de la masse calcaire [E. Boegan 1938 - figure 2], qui devrait expliquer le creusement de cheminées par la surpression lors des crues. Or ces cheminées se poursuivent toujours vers le haut, au moins par une diaclase, ce qui remet en cause la différence de pression, puisque celle-ci s'annule beaucoup plus aisément dans l'air que dans l'eau ; et ces cheminées ont suscité diverses autres hypothèses. 


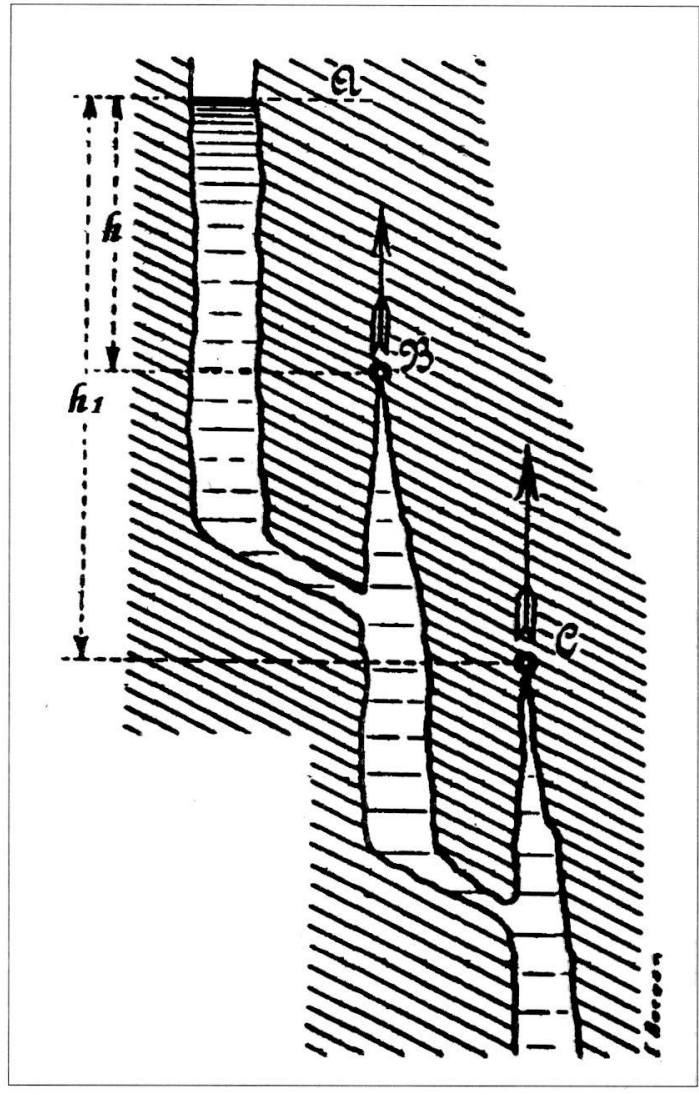

Fig. 2 - Schéma d'une série de puits, dans la grotte de Trebiciano (Italie), montrant le processus de corrosion et d'érosion de l'eau remontante et la valeur de la pression hydrostatique exercée par l'eau aux points $B$ et $C$ ( $A=$ hauteur atteinte par l'eau du fleuve souterrain) (d'après BOEGAN, 1938).

(Diagram of a series of pits in Trebiciano Cave, Italy, showing the process of corrosion and erosion af ascending water and the value of hydrostatic pressure on points $B$ and $C$ ( $A$ is the highest level reached by the water of the underground river)

En fait la pression ne peut agir par elle-même : des mises en charge correspondant de manière fréquente à des pressions de plusieurs centaines de kilopascals ne suscitent par elles-mêmes aucun désordre dans les cavités ; c'est le déplacement rapide de

l'eau et de sa charge solide qui créent éventuellement de tels désordres.

\section{Creusement par variation de pression de l'eau}

Entre deux points, les différences de pression pourraient-elles alors agir en accélérant les circulations ? Dans un réseau de fentes, les résistances hydrauliques limitent de telles accélérations. Et, dans des conduits, où les différences de pression se transmettent aisément, elles demeurent faibles. Un « réseau anastomosé » [A. Palmer 1975] ne peut donc être imputé à la pression. L'utilisation de surfaces de discontinuité, sans qu'aucune prédomine, se comprend mieux en eau stagnante, donc en l'absence de pression.

On a parfois invoqué des « alternances de pressions et de dépressions... d'où résultent tantôt de véritables explosions, tantôt des implosions désagrégeant profondément les roches » [B. Gèze 1973] ; de tels phénomènes brutaux devraient laisser des traces dans le karst profond. Or les variations de pression y demeurent relativement 
peu rapides (quelques dizaines de mètres de hauteur d'eau par heure dans la Luire, Drôme).

Pour créer le « porche d'explosion » de Martel [1930], vaste par rapport aux conduits qui le précèdent, on peut invoquer le phénomène du coup de bélier. Mais de tels porches sont souvent explicables par les processus résultant du contact des conditions climatiques extérieures avec le microclimat souterrain ; et des porches tout aussi vastes se trouvent dans des pertes, par exemple celle de Réveillon (Lot).

Pour J.N. Salomon [1986], la formation des dolines d'effondrement du Mikoboka (Madagascar) résulte d'une surpression : les forts contrastes saisonniers de pluviométrie provoquent des variations de niveau de la zone noyée, « les plafonds des salles sont soumis à d'énormes différences de pression hydraulique. C'est pourquoi les effondrements sont fréquents ». Pour l'auteur, la doline d'effondrement en chaudron serait alors « caractéristique des karsts tabulaires des régions tropicales à fort contraste saisonnier et à longue saison sèche... Des formes similaires sont également très fréquentes dans les karsts de Nullarbor en Australie où elles sont héritées [J.N. Jennings et D.C. Lowry, 1974] et dans le karst du Yucatan (cénotes) [J. Corbel 1958] ».

Sous une surface très horizontale et un plafond étanche (il peut l'être à Nullarbor), une surpression résultant d'une crue est vraisemblablement susceptible de provoquer un effondrement. Mais le plafond ne semble pas étanche dans les zones où s'ouvrent les cénotes, à Cuba. Du reste, le gouffre et la doline d'effondrement s'expliquent aussi par l'élargissement d'un conduit souterrain, tel qu'en fonction des caractéristiques mécaniques de la roche, son plafond n'est plus stable [W.E. Davies 1951].

\section{Creusement par cavitation}

Au sens physique, la cavitation est la formation de bulles de vapeur au sein d'un liquide en mouvement lorsque la vitesse atteinte est telle que la pression du liquide devient inférieure à sa tension de vapeur. Dans un conduit artificiel, il en résulte des formes comparables aux coups de gouge. Est-ce que, dans les grottes, ceux-ci ne résulteraient pas d'un phénomène de cavitation ? [Hjulström 1935].

Ph. Renault [1961] admet que la cavitation peut se produire dans un conduit si :

$$
\mathbf{V}^{2}>2 \mathrm{~g} \times(\mathbf{p}+\mathbf{h})
$$

la tension de vapeur est assez faible pour qu'on puisse la négliger aux températures ordinaires et où : 
$\mathbf{V}=$ vitesse d'écoulement $\mathrm{m} / \mathrm{s} \quad \mathbf{g}=$ accélération de la pesanteur $9,81 \mathrm{~m} / \mathrm{s}^{2} \quad \mathbf{p}=$ pression atmosphérique en mètres d'eau (avec 1 atmosphère $=10,3$ mètres d'eau) $\mathbf{h}=$ pression locale piézométrique en mètres d'eau.

Comme on a toujours $\mathbf{p}+\mathbf{h}>\mathbf{1 0 , 3}$, pour permettre la cavitation, il faudrait que la vitesse de circulation soit au minimum $V>\mathbf{1 4} \mathrm{m} / \mathrm{s}$. Les vitesses connues de circulations karstiques sont d'ordinaire très inférieures à ce chiffre : se rapprochant sans doute de vitesses instantanées, les plus petits coups de gouge connus, de 1 à 2 centimètres de long, correspondent respectivement à des vitesses de l'ordre de 4 à $2 \mathrm{~m} / \mathrm{s}$ [R. L. Curl 1966]. Le creusement par cavitation ne serait donc pas crédible [J. Corbel 1963].

Mais, dans le réseau des Siebenhengste (Suisse), une crue aurait progressé avec une vitesse locale de $50 \mathrm{~m} / \mathrm{s}$ [J.P. Bartholeyns 1987]. Encore faudrait-il trouver les marques d'une éventuelle cavitation [A. Bögli 1980]. Or si les effets de la cavitation dans des conduites en métal sont connues, " nous ne connaissons pratiquement rien actuellement de ses effets sur la roche » [A.A. Cigna 1983].

\section{Vitesse de creusement des conduits}

Trois approches ont été pratiquées pour connaître le rythme de creusement des conduits :

1) J. Coward [1971] mesure un retrait des parois de l'ordre de 1,2 millimètres par an dans la Lower Hugues Cave (West Virginia, USA) après une « correction en fonction du rapport annuel », qu'il n'explicite pas. Et quelle est la part de l'érosion mécanique? [A.N. Palmer 1981]. D'autres creusements très rapides ont été mesurés dans des karsts littoraux des Bahamas [J. E. Mylroie et J. L. Carew 1987] : dans ce cas, l'eau des circulations est chargée en chlorures, ce qui fait intervenir l'effet-sel, et d'autre part la roche est poreuse. Des creusements peuvent donc être rapides dans des conditions particulières.

2) Dans la grotte de Meyraguet (Lot), une date (1898) peinte au goudron protège la paroi (figure 3); on mesure que le recul de celle-ci, au bout d'un siècle, est de 1 centimètre. À l'endroit où se trouve cette date, la section de la grotte s'est alors accrue en moyenne de $1 / 10000^{\mathrm{e}}$ par an.

À partir de cet ordre de grandeur ou de calculs théoriques, plusieurs auteurs supposent implicitement que l'agrandissement actuel du conduit fut le même dans le passé depuis le début du creusement: c'est parfois en utilisant un modèle en deuxdi- 


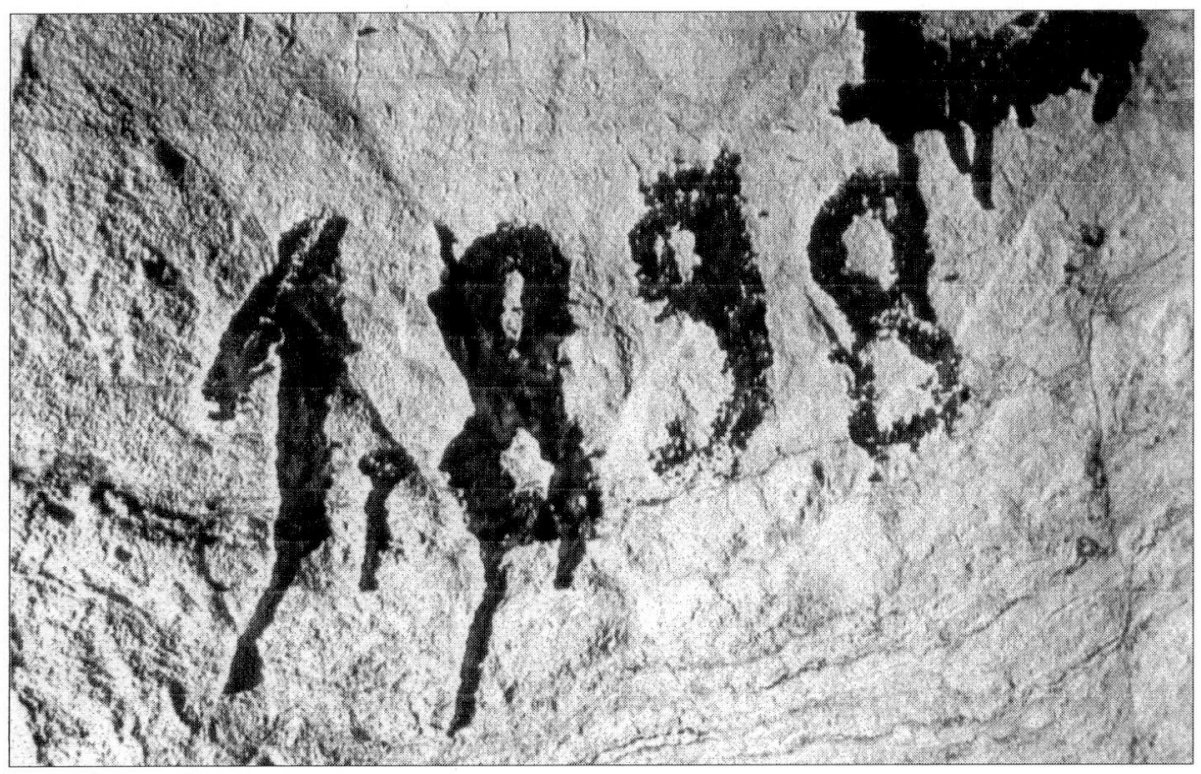

Fig. 3- Grotte de Meyraguet (Lot), inscription de paroi (Writing on the cave wall)

mensions, donc avec une extension infinie dans la troisième. Ils aboutissent ainsi à des valeurs de l'ordre de 5000 à 20000 ans, voire beaucoup moins [A.N. Palmer 1981] pour des conduits de section métrique.

Or, en volume, cet élargissement est évidemment très faible au début puisque le débit d'eau admis par le conduit, et susceptible de dissoudre, est lui-même très faible. Et, souvent, ces calculs ont été faits avec des hypothèses optimistes, par exemple en supposant des circulations initiales telles que cela revient à supposer que le creusement des conduits a déjà débuté.

3) Par bilan de matière au niveau des eaux d'un karst, on peut estimer le rapport des quantités de carbonates exportées au volume de vides karstiques ; mais ce dernier, en particulier, n'est connu que de manière très hypothétique. Dans le cas du bassin expérimental du Baget (Ariège), le calcul, toujours mené comme si l'agrandissement annuel était constant dans le temps, aboutit à des durées du même ordre de grandeur que ci-dessus [M. Bakalowicz 1979].

\section{Approche thermodynamique}

Le karst est parfois considéré comme un système " ouvert », caractérisé par des processus irréversibles d'échanges avec l'extérieur de matière et d'énergie ; son 
entropie tendant à croître jusqu'à l'achèvement de la structuration des circulations [A. Mangin 1982]. Par contre, pour P.Y. Jeannin [1996], la structuration des circulations est significative d'une réduction d'entropie, puisque les échanges se font essentiellement par consommation de l'énergie potentielle de l'eau et perte de matière [J.J. Delannnoy 1997]. Cependant, « dans un karst, il y a une entropie liée à la température de la roche (on sait la calculer et elle est très grande), et une entropie d'organisation (on ne sait pas la calculer et elle est sans doute très faible » [B. Lismonde 2000].

\section{Hypothèses relatives à l'hydrogéologie}

\section{Existence d'un karst noyé}

Certes, « l'absence de nappe dans les calcaires », si souvent défendue par Martel [1921] même s'il admettait des exceptions [J. Choppy 1997], n'est plus crédible. Mais que peut-on dire de cette nappe?

« Il semble qu'en matière de spéléogenèse tous les auteurs sont d'accord sur la présence d'un karst noyé » [MANGIN 1975].

Sur la présence du karst noyé, oui. En matière de spéléogenèse, c'est moins évident !

1) Il est vrai que les conduits noyés, parfois sur une grande profondeur, sont légion. Et sous le niveau de base, à peu près tous les vides, quelle que soit leur origine, sont pleins d'eau. Mais l'existence actuelle de vides karstiques noyés n'implique pas qu'ils l'aient toujours été.

2) On verra ci-dessous qu'une morphologie considérée comme caractéristique d'un régime noyé ne l'est peut-être pas.

3) Des phénomènes qui n'étaient pas toujours mis en relation avec le karst, autrefois, se développent effectivement en régime noyé : karst hydrothermal, karst artésien, creusement supposé à la limite eau salée - eau douce.

L'existence de cavités noyées a tout naturellement conduit à penser que leur creusement s'est fait en régime noyé.

\section{Hypothèse d'une nappe phréatique occupant les conduits}

O. Lehmann [1932] représente les circulations du karst comme une nappe phréatique 
occupant les conduits, soumise à un jeu de vases communicants, à laquelle se superposent quelques écoulements torrentiels (figure 4).

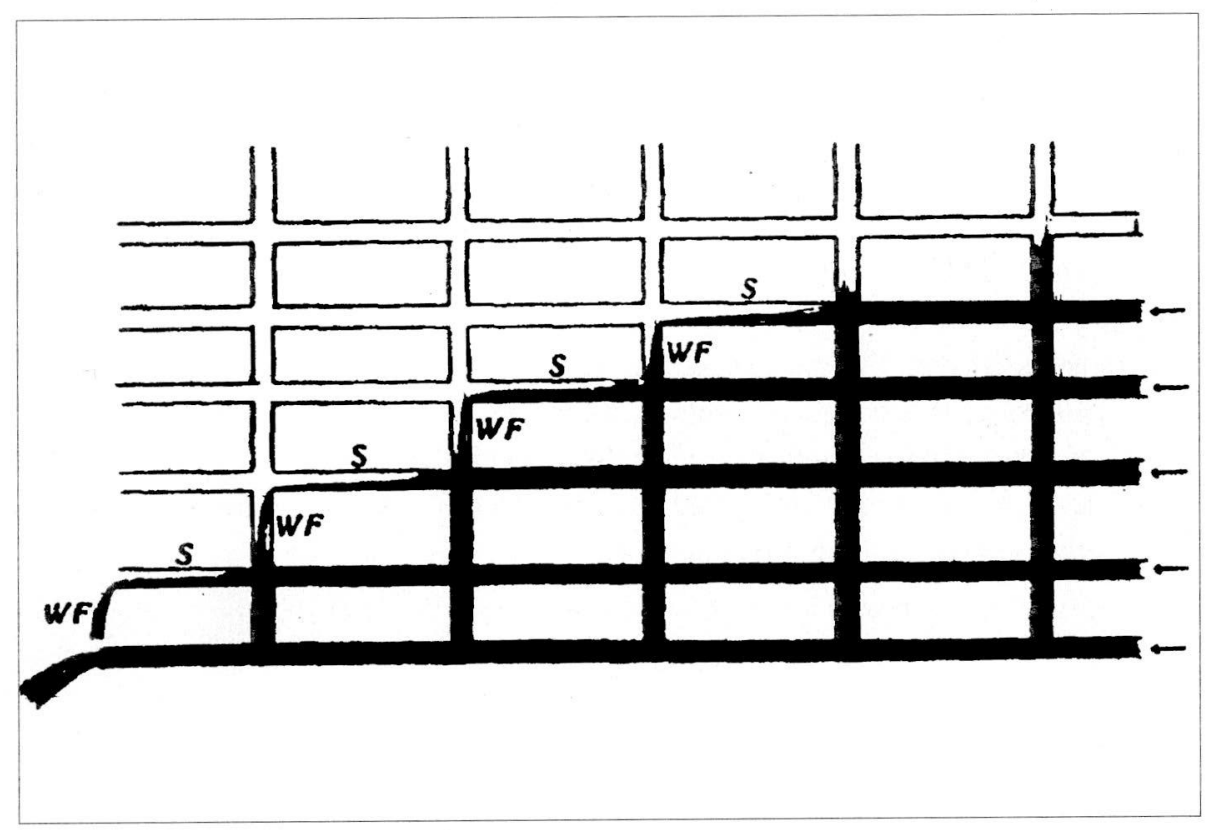

Fig. 4 - Schéma des circulations dans le karst. $S=$ circulation en écoulement libre, $W F=$ cascade (d'après LEHMANN, 1932) (Diagram showing water drainages in karst.

$S=$ vadose drainage, $W F=$ waterfall $)$

La considérable perméabilité des massifs karstifiés, encore plus importante dans le réseau de conduits, qui semblait alors inconnue, exclut des nappes aussi pentées. Et ce système n'inclut pas les nombreuses curiosités hydrauliques du karst : relations entre réseau de fentes et conduits, circulations suspendues, superposées, etc. Ce schéma fait plutôt penser à un ennoyage, ce dont des preuves existent dans le karst slovène.

\section{Hypothèse d'une organisation en drain et systèmes annexes}

L'amont de la source intermittente de Fontestorbes (Ariège) a été trouvé dans l'aven des Caoujous, distant de 1120 mètres, sous forme d'oscillations de plans d'eau de même période que les intermittences de Fontestorbes. Mais la montée de l'eau est plus lente dans l'aven des Caoujous que la descente, alors que c'est l'inverse à Fontestorbes. Cela ne semblerait pas illogique si l'aven des Caoujous se situait en 
amont du mécanisme d'intermittence. On ne peut le savoir car, si la circulation alimentant l'aven des Caoujous, qui ne présente pas d'intermittences, a pu être explorée [Hernandez 1984], son débit est beaucoup plus faible que celui de Fontestorbes [A. Mangin 1995].

Or, si l'aven des Caoujous est en aval du mécanisme d'intermittence, les « réactions du karst » entre celui-ci et l'exutoire sont surprenantes. Cela conduit A. Mangin [1969] à proposer l'hypothèse d'un drain, aboutissant à l'émergence, et de « systèmes annexes » organisés de manière ramifiée. Les «drains », conduits parcourus par des circulations, se comportent « comme des réservoirs peu capacitifs mais transmissifs »*; ils drainent des « systèmes annexes », « réservoirs très capacitifs, mais peu transmissifs dont ils assurent en outre les seules liaisons ». Ces systèmes annexes sont « le siège d'écoulements lents »; ils ne sont pas « en relation les uns avec les autres, mais tous en relation avec le drain ». Et, à l'intérieur d'un système de $2^{\mathrm{e}}$ ordre, des «systèmes de $3^{\mathrm{e}}$ ordre sont en relation les uns avec les autres et l'un d'eux est en relation avec le drain » (figure 5).

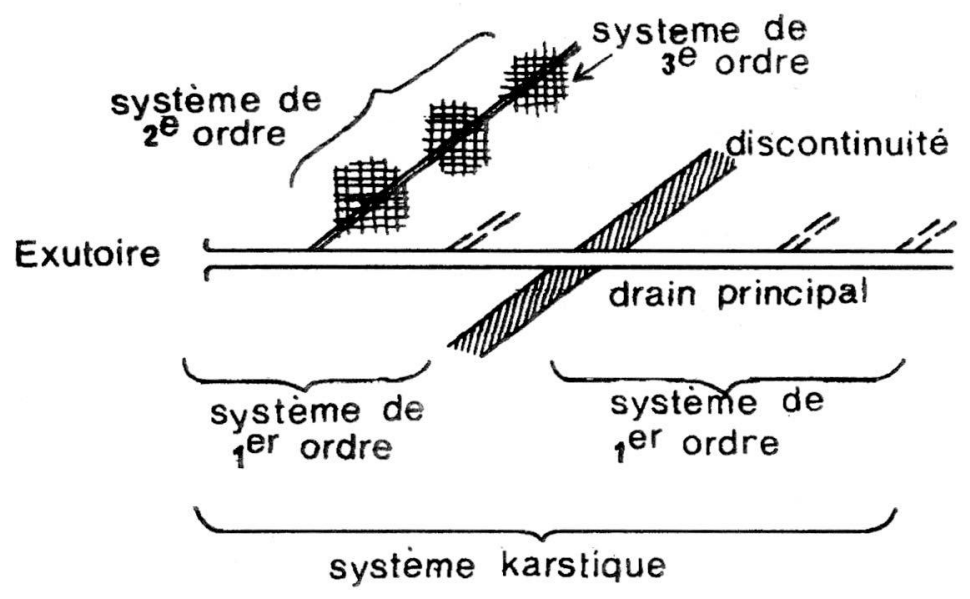

Fig. 5 - Représentation schématique d'un système karstique (d'après MANGIN, 1969) (Schematic diagram of a karst system $»$ Exutoire $=$ water outlet $)$

«Les systèmes annexes ne sont absolument pas synonymes de réseau de fentes et de fissures. Il existe à leur niveau des cavités de grandes dimensions (ainsi, sur le Baget,

* capacitif = qui peut contenir de l'eau ; transmissif = qui assure la circulation de l'eau. 
des galeries de 4 mètres de diamètre sont situées dans le système annexe de la Peyrère, Ariège) ». Les circulations entre les systèmes annexes et les drains « peuvent s'effectuer avec de fortes pertes de charge (cas du système annexe du secteur de P15 sur le Baget) ou avec de faibles pertes de charge (cas du système annexe de la Hillère sur le Baget, Ariège) » [Mangin 1975].

On peut se demander :

1) Comment une circulation peut-elle, avec une faible perte de charge, présenter une faible transmissivité ?

2) Comment une organisation hiérarchisée en drains et systèmes peut-elle se mettre en place ? Car les phénomènes susceptibles de réduire ou d'altérer la transmissivité, des colmatages par exemple, sont aléatoires dans un karst.

3) Il ne semble pas qu'on ait jamais observé une morphologie souterraine dont le fonctionnement hydraulique puisse être en drain et systèmes annexes.

\section{Étude des courbes de crue-décrue}

Par l'étude de la courbe de décrue-tarissement au niveau d'une émergence, les hydrogéologues cherchent à apprécier l'importance du réseau de fentes et du réseau de conduits. Il apparaît d'ordinaire que le volume du premier est nettement le plus important, mais la méthode les distingue de manière relativement arbitraire : des conduits isolés par un bouchon de sédiments peu perméables seront compris comme des fentes. Et les vides qui ne sont pas atteints par la crue sont ignorés

L'étude de ces mêmes courbes par analyse corrélatoire et spectrale a conduit A. Mangin [1982] à classer des circulations souterraines selon qu'elles restituent les précipitations avec un filtrage modeste (elles sont alors «bien drainées »), et celles qui sont au contraire «mal drainées » (figure 6).

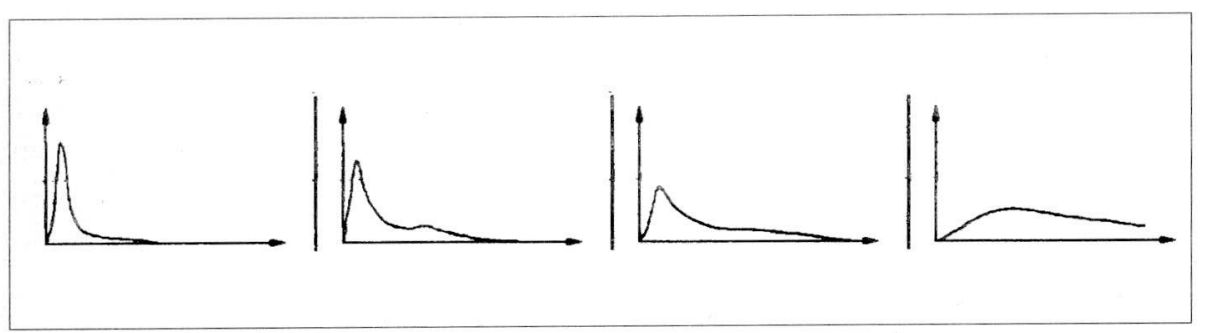

Fig. 6 - Hydrogrammes-type de circulations, à gauche « bien drainées », et de plus en plus "mal drainées » en allant vers la droite (d'après MANGIN, 1982) (Typical hydrograms showing good drainage on the left and more and more bad drainage on the right.) 
Cette classification ne s'adapte qu'en partie à d'autres systèmes :

Les hydrogrammes de la fontaine de Vaucluse (Vaucluse) peuvent être de plusieurs des types ci-dessus [J.M. Puig 1990].

- Dans le massif de la Sainte-Baume (Bouches-du-Rhône - Var) ont été retrouvés « tous les types de fonctionnement hydrodynamiques définis... à partir des analyses corrélatoire et spectrale »[Ph. Martiin 1991].

Les qualifications de «bien » ou « mal drainé », dont la définition est hydraulique, ne sont pas une mesure de la karstification. Car si la structuration progressive des circulations karstiques en améliore le drainage, d'autres causes, éventuellement transitoires, peuvent modifier l'hydrogramme.

\section{Hypothèses relatives aux facteurs}

Des «facteurs » géologiques et géographiques activent ou inhibent les processus.

\section{Rôle de la lithologie.}

«Certaines régions de l'Himalaya se distinguent par l'absence de grottes dans le calcaire, parce que la roche a été déformée à l'état plastique et n'a donc pas été fracturée pour former des séries de diaclases à partir desquelles les grottes auraient pu se développer » [A.C. Waltham 1971]. Pourtant, après avoir été déformées de la sorte, ces roches ont été portées à l'affleurement par une surrection génératrice d'une néofracturation ; la rareté des cavités, effectivement observée par d'autres auteurs, devrait avoir d'autres causes.

On sait qu'en tant qu'aquifère, une roche est capacitive en raison de sa porosité (efficace) ; mais elle n'est transmissive qu'en fonction de sa perméabilité.

\section{Rôle de la tectonique}

Depuis que l'on sait que la surrection est souvent supérieure à l'ablation karstique [M. Julian 1992], l'hypothèse d'un cycle karstique [A. Grund 1914], qui suppose un relief essentiellement généré par des processus karstiques (figure 7), déjà contestée par J. Roglic' [1974], a perdu de sa crédibilité. Il en est de même de la « surface d'aplanissement karstique », dans la mesure où elle est supposée constituer l'aboutissement de ce cycle. 


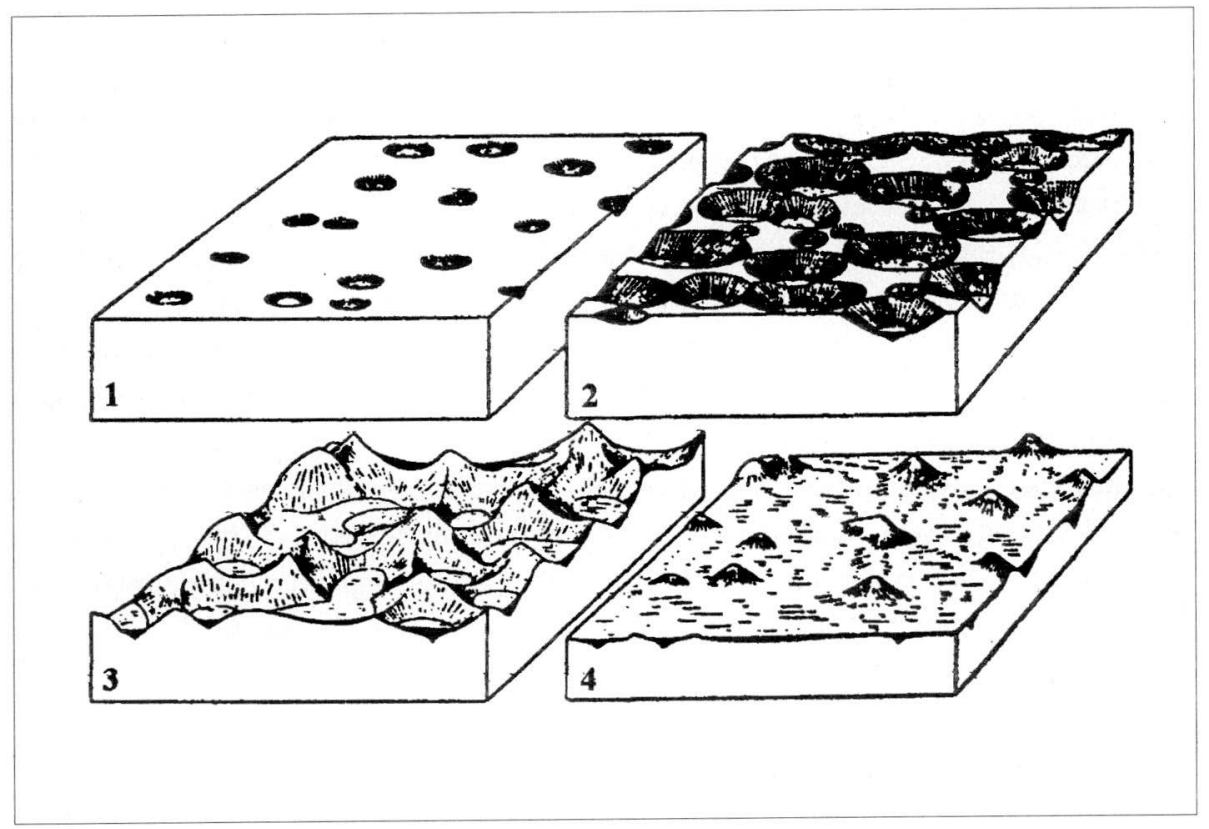

Fig. 7 - Schéma du cycle karstique:

1. Karst jeune, dans les larges surfaces planes entre les dolines, la forme initiale est encore reconnaissable.

2. Karst adolescent, les dolines sont devenues plus grandes et nombreuses et sont pour une part déjà coalescentes.

3. Karst mûr (karst à buttes), la forme initiale est disparue, de grandes dolines et ouvalas se trouvent entre les buttes.

4. Karst vieux (karst à buttes), entre les buttes, le pays est aplani, les buttes se dressent encore en collines basses isolées au dessus d'une surface résiduelle (d'après A. GRUND, 1914; dans la traduction française de la légende de cette figure, la confusion faite par l'auteur entre cockpit et butte [karstique] a été corrigée)

(Model of the karst cycle:

1. Young karst: In the extensive flat areas between the dolines, the original surface is still recognisable.

2. "Adolescent" karst: The dolines have become larger and more numerous, and have already begun to coalesce.

3. Mature karst ("cokpit" landscape): The original surface has disappeared; large dolines and uvalas lie between the "cockpit" hills.

4. Old karst ("cockpit" landscape): Between the "cockpit" hills the land has become evened out. The hills, now isolated and low, still stand out above the residual plain that coming into being.

In the English translation, the confusion made by the author between cockpit and [karst] cone has not been corrected, but the word cockpit is in quotation marks) 
En cas de pendage élevé, l'eau pénètre aisément en profondeur, mais on a pu croire que sa concentration demeure médiocre, conduisant à un « déficit presque mondial de grottes » [A.C. Waltham 1971]. La même affirmation fut faite pour les massifs de l'Alpette et du Granier (Chartreuse, Savoie) : lorsque le pendage excède $40^{\circ}$, « le bassin versant est peu étendu; il y a donc peu d'alimentation et pas de creusement possible » [Anonyme 1964]. Les explorations faites depuis dans le Granier ont révélé un important kilométrage de conduits souterrains. L'idée d'un rôle négatif du pendage, qui résulte d'une image simplificatrice des surfaces de discontinuité géologiques est encore contredite par de grandes grottes comme le Hölloch (Suisse) ou Han-sur-Lesse (Belgique).

De plus, les conduits qui se développent en «méprisant » apparemen les fractures et joints de stratification, sont innombrables. En fait, ces surfaces de discontinuité ne « contrôlent» (« control » des publications anglophones) pas les circulations karstiques. Au contraire, celles-ci les utilisent selon leur propre logique.

\section{Corrélations entre phases tectoniques et creusement karstique.}

La tentation est grande d'admettre que des phases de creusement dans le karst sont en relation avec des événements tectoniques ; la corrélation étant même assez précise entre ceux-ci et le démarrage de la karstification pour pouvoir dater l'un par l'autre [J. Demangeot 1965].

On a donc cherché à reconnaître dans les directions de conduits celles des fractures créées par la phase tectonique, d'abord par observation directe [H. Tintant 1958 ; L. Kiraly 1968]. Ultérieurement R. Guérin [1973] procède par voie statistique, en mesurant les directions de conduits souterrains sur les plans de cavités. Cette démarche fut critiquée par S. Puyoo [1976], Y. Callot [1979], A. Eraso et al. [1983], car :

- les fractures visibles dans les grottes, même des failles, ne se retrouvent pas nécessairement en surface [C. Ek 1970];

- les galeries qui figurent sur les plans spéléologiques sont majoritairement établies sur des joints de stratification et non des fractures.

Dès lors, considérer les directions de conduits comme comparables aux seules directions de fractures est une pétition de principe ; de toutes façons l'observation sur place demeure indispensable. Et il est difficile de préciser l'époque d'utilisation d'une fracture ayant rejoué au cours de diverses phases tectoniques. 
Par une démarche inverse, on cherche à prédire le trajet des circulations souterraines à partir d'observations en surface, soit de fractures [S.E. Lauritzen 1989], soit pour rétablir les ellipsoïdes de contraintes [A. Eraso 1986]. Cette deuxième approche prend en compte le relief, la localisation des émergences, et l'on ignore ce qui contribue aux résultats spectaculaires annoncés. Ceux-ci ne se confirment pas quand la méthode est appliquée au réseau des Siebenhengste (Suisse - P.Y. Jeannin 1989]. L'effet d'une phase tectonique sera plutôt de créer une charge hydraulique ; mais cela n'entraîne pas immédiatement le creusement de conduits : intervient d'abord un temps de latence durant le creusement d'un réseau de fentes, encore plus long s'il doit comprendre le dégagement d'une couverture. Et d'innombrables circulations suspendues montrent qu'un retard dans l'adaptation des circulations souterraines et aériennes est habituel, même avec une charge hydraulique importante.

\section{Rôle du climat et du microclimat}

À une époque où le rôle du climat était considéré comme le facteur essentiel déterminant les morphologies, H. Lehmann [1960] a défendu énergiquement l'idée que les formes des karsts de régions chaudes ne pouvaient être décrites avec les noms attribués aux formes tempérées.

Il faut en particulier revenir ici sur le karst à buttes, longtemps considéré comme une forme tropicale. Or des karsts à buttes plus ou moins frustes existent dans beaucoup de régions tempérées, et jusque dans le karst «classique » de Slovénie. Ce peut être un héritage. Mais si le karst à buttes est mieux développé dans certaines zones climatiques, il ne s'y développe pas toujours ; tandis qu'une influence tectonique est parfois indéniable [S. Sebela 1998]. Et l'on a vu qu'une surrection pourrait y contribuer.

Dans un autre contexte, des cavités « cutanées » furent attribuées au creusement en conditions périglaciaires dans une couche superficielle de terrain, lors du dégel estival [R. Ciry 1959]. Pendant ce délai, compte tenu de la conductivité thermique du calcaire et de la chaleur latente de fusion de la glace, on ne peut escompter que le dégel dépasse un ou deux mètres d'épaisseur de roche, pour une durée de quelques semaines. Et, comme la dynamique de réaction est réduite à basse température, un tel creusement aurait nécessité des conditions périglaciaires stables pendant des centaines de milliers d'années ; ce qui semble ne jamais avoir existé dans les régions où sont décrites les cavités cutanées.

Dans la région de Mo I Rana (Norvège), plusieurs cavités semblent avoir été des dérivations souterraines de bédières [G. Horn 1947]. J. Corbel [1957] pensait que 
l'eau provenant d'un glacier ne creuse pas dans la roche, ce que contredit la grotte de Gébroulaz (Savoie), dans le gypse.

Et la spécificité de formes "climatiques » fut contestée [G.A. Brook, D.C. Ford 1976]. Certains aspects spectaculaires de karsts chauds sont-ils dus au climat ou seulement à une karstification qui s'est poursuivie sans avatar pendant une longue durée ? Du reste, si la distinction entre formes froides, tempérées, tropicales humides était évidente, il y a longtemps que nous disposerions d'un catalogue !

Parmi les hypothèses proposées pour expliquer le chenal de voûte, certaines furent micro-climatiques. En effet, des chenaux de voûte de section centimétrique furent d'abord décrits en langue allemande, comme résultant d'un Wirbelbewegung (= mouvement tourbillonnaire) de l'eau dans un plafond de gypse [H. Cramer, F. Heller 1935 - figure $8 \mathrm{a}]$.

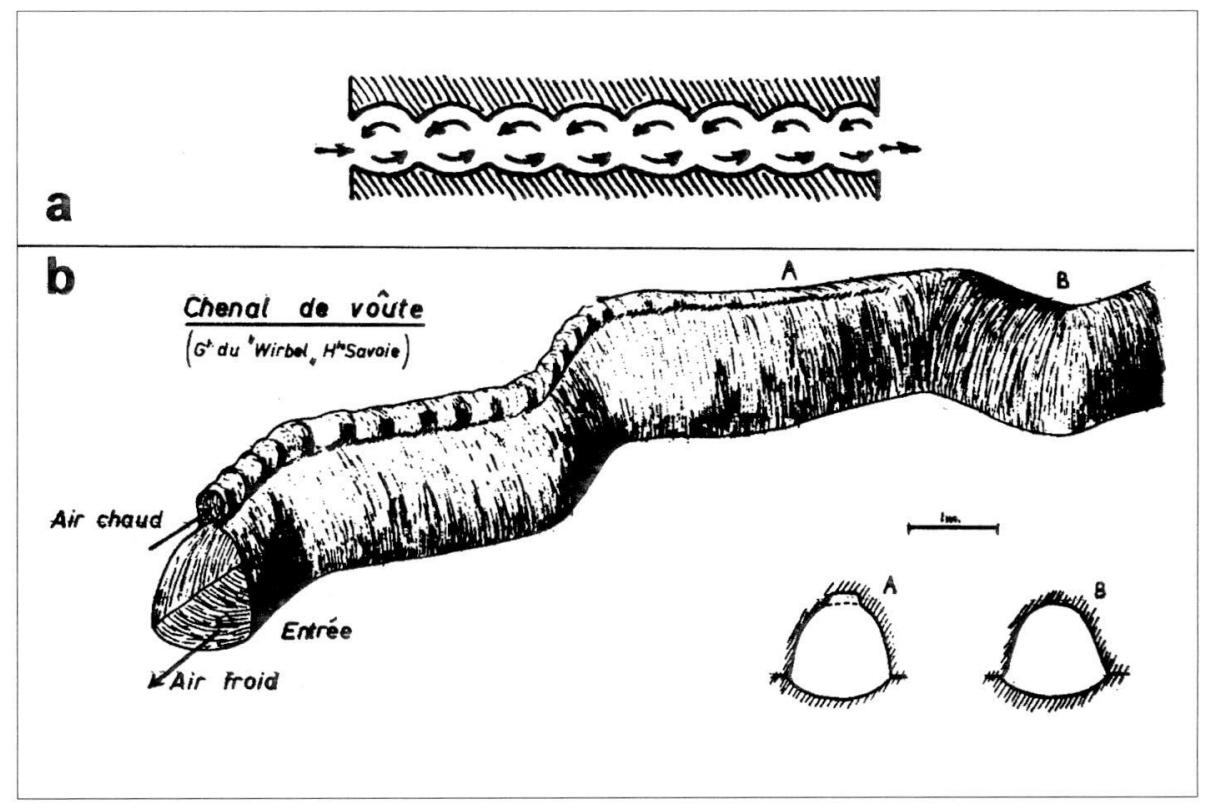

Fig. 8a - Dans le gypse de Franconie (Allemagne), vue schématique du mouvement tourbillonnaire de l'air (= Wirbel en allemand) dans un conduit lié à un joint de stratification (d'après CRAMER, HELLER 1935) (In the Franconian gypsum (Franken), Germany, schematic view of whirlwind (= Wirbel in german in a passage linked to a bedding plane.)

Fig. $8 b$ - Grotte du «Wirbel» (Haute-Savoie), vraisemblablement dénommée par assimilation, vue cavalière de la zone d'entrée. "Les chenaux de voûte ne s'observent qu'au voisinage des entrées. En profondeur ils s'atrophient en s'élargissant et se fondant à la voûte... en été l'air chaud entre par le haut de la galerie et de l'eau se condense. Il y a alors corrosion. L'air froid ressort par le bas ». (d'après MARTINI, 1960) (Grotte du Wirbel, Haute-Savoie, France. The 
name probably comes by assimilation. View of the entrance zone. "Ceiling channels can only be seen near the entrance. Deep in the cave, they degenerate, get larger, and tend to merge into the ceiling itself... In summer warm air enters the cave by the top of the passage and water condenses. Then there is corrosion. Cold air gets out at the bottom of the cave)

Puis J. Martini [1960 - figure 8b] décrit le chenal de voûte (traduction de l'expression anglaise « ceiling channel» que l'on trouve dans J.H. Bretz [1956]) comme se rencontrant seulement dans des entrées de grottes.

Certes, quand des dépôts glaciaires obturent une entrée de cavité (exemple de la Balme de Cluses, en Haute-Savoie), l'eau va plutôt creuser dans la voûte pour ressortir au jour, mais ce n'est pas exclusif. Car les témoins de sédiments limitant la base du chenal de voûte sont assez nombreux, même loin des entrées, pour attribuer celuici à un creusement ascendant [Ph. Renault 1958]. Un tel surcreusement à partir d'un « tube » initial est du reste admis par J.H. Bretz [1942].

\section{Hypothèses sur le début de l'évolution karstique}

\section{Le tout premier développement}

Pour C. Mégnien [1964], le creusement du réseau de fentes se fait en écoulement laminaire, tandis que les conduits se creusent en écoulement turbulent. Cette hypothèse est reprise par des anglophones* comme S.R.H. Worthington [1991], C.G Groves et A.D. Howard [1994], chez qui le « early karst system development » est en pratique le creusement du réseau de fentes. Mais ces auteurs notent que, dans les deux types d'écoulements, la vitesse d'accroissement des vides karstiques ne peut être calculée, compte tenu de tous les éléments entrant en jeu : «le débit et la composition de l'eau, la géométrie comme la largeur des passages et leurs relations avec leur alimentation et leur évacuation » [C.G Groves et A.D. Howard - 1994] ; ils s'en remettent à une modélisation pour comprendre comment les circulations évoluent.

Le modèle qu'ils proposent pour l'écoulement laminaire (figure 9) comporte une charge hydraulique de 40 mètres pour une longueur de 750 mètres entre l'entrée et la sortie, soit une pente d'environ $5 \%$ sur l'horizontale. Les vides ont un diamètre initial de 1 millimètre. Le diamètre final maximum est de l'ordre de 7 millimètres à l'entrée, et diminue vers la sortie, approximativement selon une fonction exponentielle négative. Le débit de sortie est multiplié par environ 60

\footnotetext{
* Les hypothèses sur le creusement karstique développées par des auteurs anglophones ont été reprises dans National Speleological Society [2000].
} 
pendant la durée de fonctionnement (qui, par effet d'échelle, correspond à 4100 années).
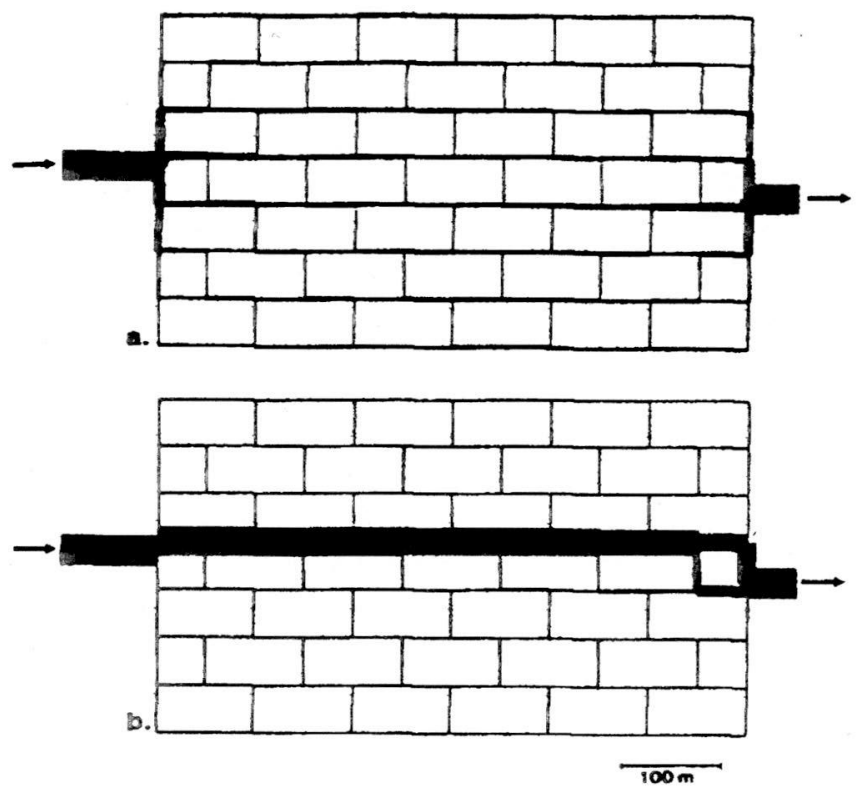

Fig. 9 - Vue en plan de l'essai 1 montrant les débits initial (a) et final, c'est-à-dire au début de l'écoulement turbulent (b) (d'après GROVES, HOWARD 1994 (Section view of run 1 showing (a) initial and (b) final discharges at onset of turbulence).

Ce modèle présente une entrée d'eau concentrée et débouche sur une sortie unique (un autre modèle, mis en oeuvre par ces auteurs, comporte plusieurs entrées identiques superposées). Pourtant, au début du développement karstique, la pluie tombe sur un massif encore indemne de toute karstification, et l'eau ne dispose pas davantage d'une sortie unique. De plus, malgré la faiblesse des diamètres, il n'est pas question de capillarité dans ce modèle.

Plus généralement, P.Y. Jeannin [1996] met l'accent sur l'écart entre les circulations karstiques observées et les modèles destinés à les représenter. Cet écart apparaît plus grand encore dans la mesure où ces modèles s'inspirent d'hypothèses qui sont discutées ici. Et faut-il rappeler qu'un modèle n'est pas une démonstration? 


\section{Les hypothèses classiques sur la karstification initiale}

Puisque la vitesse de circulation est rapide dans les conduits et lente dans les fentes [E. de Martonne 1926], le karst est le siège d'une « double circulation » [Ph. Renault 1959]. Cette notion est bien connue des hydrogéologues, car elle se vérifie aisément, le débit et le chimisme des deux circulations étant différents.

Or la plupart des auteurs ayant cherché à comprendre le début de la karstification n'ont pas tenu compte de ce qu'il fallait expliquer cette double circulation. Et ils admettent implicitement cet exposé mille fois entendu : « en traversant l'atmosphère et le sol végétal, l'eau des précipitations dissout du $\mathrm{CO}_{2}$; elle arrive agressive au contact du calcaire, en élargit les fentes ; certaines de ces fentes s'agrandissent jusqu'à devenir pénétrables à l'homme, ce sont les réseaux spéléologiques ». Autrement dit, ces auteurs supposent que les conduits se creusent sans aucun préalable.

Pourtant, si l'on doit admettre la coexistence d'un réseau de fentes et d'un réseau de conduits, on ne voit pas comment ils auraient pu se creuser en même temps : le réseau de fentes est nécessairement antérieur au réseau de conduits qui, grâce à une vitesse de l'eau plus rapide, tend à confisquer toutes les circulations. Une étude sur modèle ne prenant pas en compte cette évolution est difficilement crédible.

E.A. Martel [1921] a toujours défendu la notion de «rivière souterraine », expression qu'il faudrait expliciter car elle ne représente qu'une modeste proportion de la diversité des circulations et des formes karstiques; et il n'a pas expliqué comment pouvait se passer le début du cavernement. Mais sa pensée n'était pas aussi monolithique qu'on pourrait le croire. Car il fut l'un des premiers à décrire des formes comme creusées en régime noyé : au Mas Raynal (Aveyron - 1894), dans la grotte de Miremont (Dordogne) et dans celle de Mitchelstown (Irlande - 1897), dans la grotte de La Balme (Isère - 1899), dans le Traouc (Aveyron - 1930). De même, chez J. Cvijic' [1927], on trouve de loin en loin l'idée d'un creusement ayant pu se faire sous le niveau de base. Et ils sont à peu près certainement les premiers à avoir interprété des formes pouvant y correspondre.

Par un cheminement de pensée dont on trouvera l'historique chez J. Roglic' [1972], A. Grund [1903] aboutit à l'hypothèse que les conduits karstiques se sont creusés dans la zone saturée. On verra également comment W.M. Davis y a adhéré, mais cela est surtout explicite dans sa publication de 1930, où il intègre de façon claire les cavités noyées dans une hypothèse sur l'origine des cavernes calcaires. Adaptant un schéma de F.H. King [1899] relatif aux roches à perméabilité d'interstices, W.M. Davis [1930] fait l'hypothèse d'un creusement débutant en régime noyé ( «phreatic»- figure 10) selon des trajets courbes, et se poursuivant en écoulement libre («vadose ») après une descente du niveau de base. 


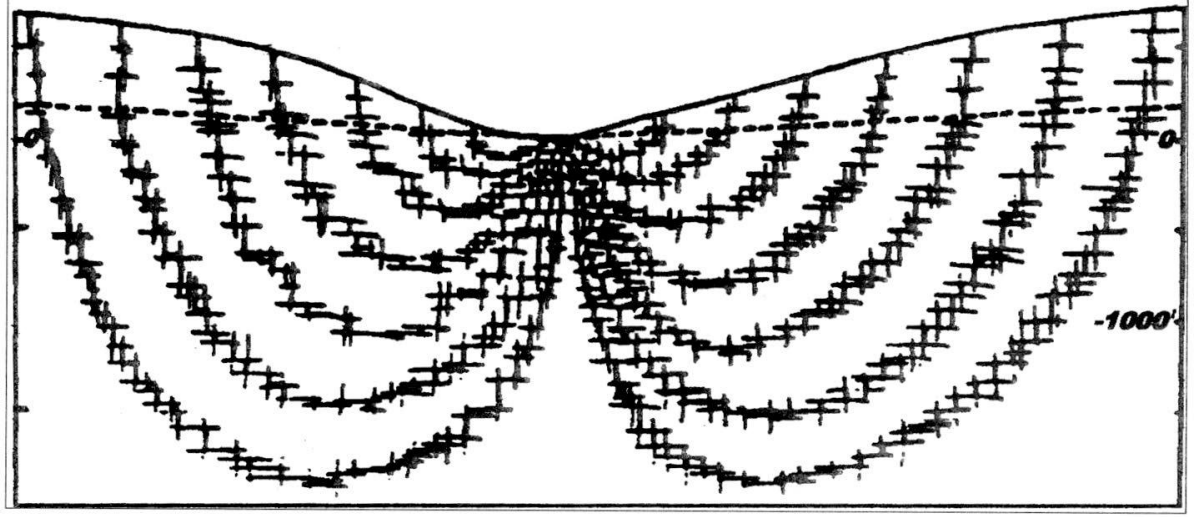

Fig. 10 - Vue en élévation de quelques-unes des lignes de courant courbes supposées dans les circulations karstiques, avec élargissement des surfaces de discontinuité géologiques; la ligne en tireté représente la surface piézométrique ; échelle verticale en pieds (d'après DAVIS, 1930) (Cross section of a few supposed curved current lines in karst drainages, with a widening of geologic discontinuities. The dotted line is the piezometric surface).

Selon A.C. Swinnerton [1932], les rôles joués par les diverses lignes de courant courbes ne sont pas identiques, car « la circulation de l'eau dans les roches est analogue à un système de potentiels »; dès lors la résistance hydraulique s'accroît donc quand la ligne de courant s'allonge ; autrement dit « la direction de moindre résistance est la plus courte », c'est-à-dire la ligne droite (figure 11). Et diverses observations de terrain montrant que la densité des diaclases est décroissante avec la profondeur, Swinnerton en déduit que l'essentiel du creusement se fait « au dessus et dans la partie supérieure de la nappe phréatique ».

Ces observations sont valables même dans le cas d'une perméabilité d'interstices. Par contre, la ressemblance des lignes de courant courbes avec celles d'un « flux d'électricité » [C.S. Slichter 1899], comme dans la méthode géophysique du sondage électrique, est un faux-semblant car le déplacement de l'électricité, dans des roches moins fracturées et imprégnées d'une eau moins mobile, donc plus minéralisée, bénéficie d'une conductivité croissante avec la profondeur. Et c'est le contraire pour la circulation de l'eau. 


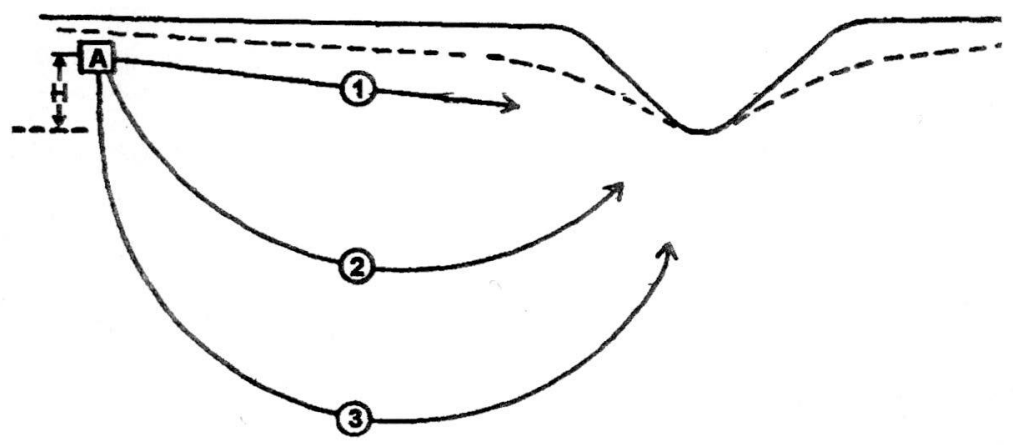

Fig. 11 - Schéma des charges hydrauliques d'une circulation d'eau. (d'après SWINNERTON, 1932)(Diagram of Water-flow Potentials. The volume of water $(A)$ is actuated by the head $(h)$ to flow in all possible paths toward the outlets. Flow is resisted in all the directions but is resisted last in the shortest path (1) most in path (3). The water (A) will divide itself into amounts proportionate to the ease of flow. Homogeneous rock and uniform distribution of joints are assumed)

Sous l'influence de la charge hydraulique $(\mathbf{H})$, le volume d'eau $(\mathbf{A})$ est conduit à couler dans tous les chemins possibles vers l'émergence. Selon toutes ces directions, une résistance hydraulique s'oppose à la circulation, mais elle est plus faible selon le chemin (1) que selon le chemin (3). L'eau (A) va automatiquement se diviser dans les mêmes proportions pour circuler aisément. On suppose une roche homogène et une distribution uniforme des diaclases », donc une conductivité constante.

Par ailleurs, le creusement en régime noyé supposé par W.M. Davis [1930] est contredit par l'application d'une loi physique : selon le théorème de BERNOULLI, dans un conduit, en l'absence de frottements, la charge hydraulique $\mathbf{H}$ (en mètres dans la formule ci-dessous) est constante en régime noyé le long d'une ligne de courant.

$$
\mathbf{H}=\mathbf{p} / \mathbf{r} . \mathbf{g}+\mathbf{V}^{2} / 2 \mathrm{~g}+\mathbf{z}=\mathbf{C t e} .
$$

avec $\mathbf{p}:$ pression $\left(\right.$ pascal $\left.=\mathrm{kg} / \mathrm{m} \cdot \mathrm{s}^{2}\right)$

$\mathbf{r}$ : masse volumique de l'eau $\left(\mathrm{kg} / \mathrm{m}^{3}\right)$

$\mathbf{g}$ : accélération de la pesanteur, soit 9 mètres/seconde ${ }^{2}$

$\mathbf{V}$ : vitesse de l'eau $(\mathrm{m} / \mathrm{s})$

$\mathbf{z}$ : altitude (m) 
Certes, selon cette formule, l'énergie dynamique d'une circulation descendante dans un conduit devrait permettre à l'eau de franchir le niveau de base. Avec les plus fortes vitesses habituelles mentionnées ci-dessus, soit environ 4 mètres/seconde, on calcule que cette possibilité n'atteint pas tout à fait 1 mètre ; bien entendu, à condition que le conduit se poursuive sous le niveau de base.

S'il n'existe pas encore de conduit, aucune circulation ne peut progresser sous le niveau de base, même avec des vitesses plus importantes. Et il ne peut y avoir de creusement, au moins dans le cas général. Enfin, à un mètre sous le niveau de base, la réduction de la viscosité de l'eau liée à son échauffement (WORTHINGTON 1991) ne peut évidemment intervenir.

Souhaitant conforter l'hypothèse d'un creusement sous le niveau de base, FOURNEAUX [1994 - figure 12] écrit : « à l'instant t0, avant l'averse, la pression aux points $\mathrm{a}$ et $\mathrm{b}$ est égale à la pression atmosphérique. Au temps $\mathrm{t} 1$, lorsque la recharge atteint le point a, la pression devient égale à $\mathrm{H}$, c'est-à-dire à la hauteur de la colonne d'eau. Mais la pression en b reste égale à la pression atmosphérique ».

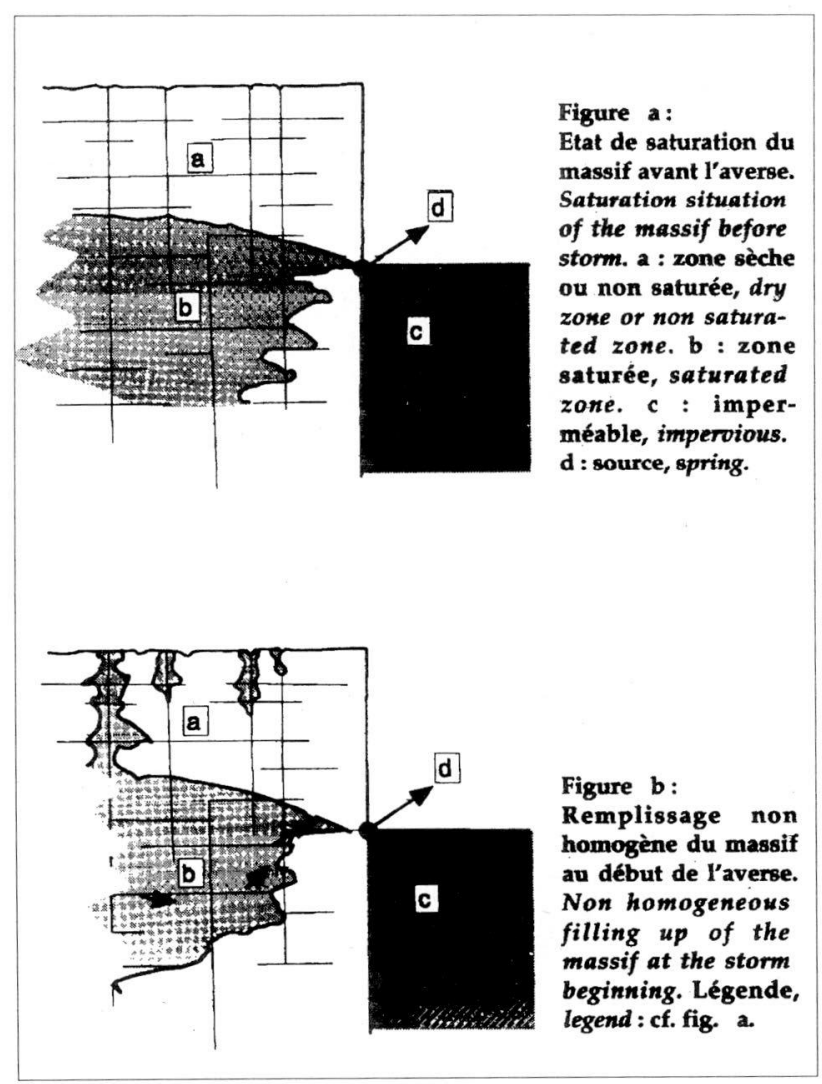

Le niveau de l'imperméable localisant la source de la figure 12 doit être considéré comme le niveau de base, donc celui d'affleurement de la surface piézométrique (représentée figure 10). Plus bas, tous les vides sont pleins d'eau et, si la vitesse de l'eau est nulle, le terme $\mathbf{p} / \mathbf{r} . \mathbf{g}+\mathbf{z}$ de la formule de BERNOULLI est constant : quand l'altitude décroît, la pression augmente. La pression dans la zone saturée est donc toujours supérieure à la pression atmosphérique.

Fig. 12 - (d'après

FOURNEAUX 1994) 
R. Rhoades et M.N. Sinacori [1941] adaptent un schéma initial selon des trajets courbes. Pour ensuite le faire coïncider avec le fait d'expérience que les conduits sont globalement pentés en direction de l'émergence, ils supposent que la dissolution se concentre progressivement dans les trajets les plus directs et dans ceux directement connectés à l'émergence. La réunion des cavités élémentaires qui se creusent ainsi le long des lignes de courant aboutirait au creusement d'un collecteur faiblement penté vers l'émergence, se développant de l'aval vers l'amont (figure 13).

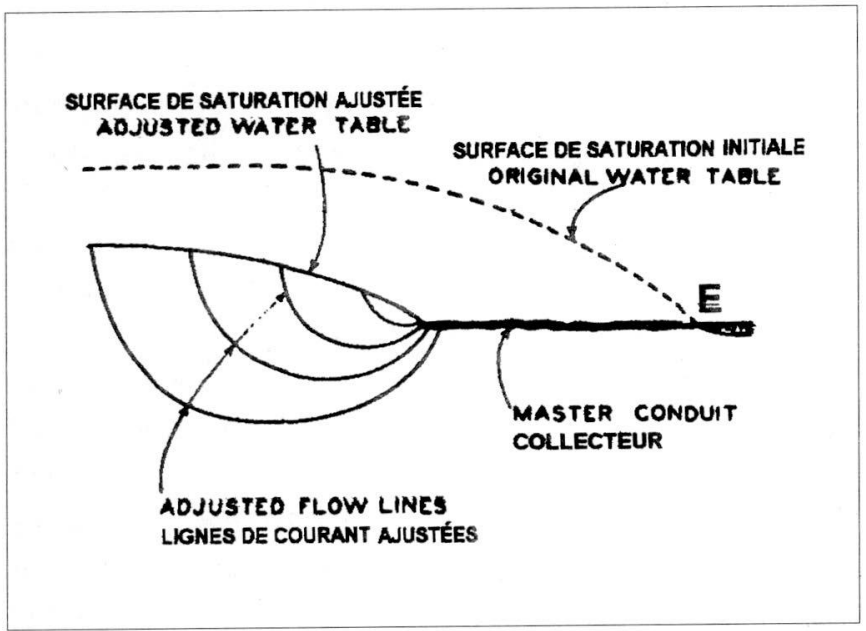

Fig.13 - Stade ultérieur de développement $d u$ collecteur et ajustement consécutif des lignes de courant ». $E=$ émergence 13 (d'après RHOADES, SINACORI 1941) (Later development of a master conduit and corresponding adjustment of lines of flow. $E=$ spring)

On peut enfin se demander si l'hypothèse d'évolution de W.M. Davis [1930], ou celles qui en sont dérivées, peuvent être considérées comme universelles. Poser la question [K.H. Pfeffer 1995], c'est déjà y répondre ! Du reste J.H. Bretz [1942] admettait que certaines cavités s'étaient creusées uniquement en écoulement libre.

\section{Aspects morphologiques des creusements «phréatique »et «vadose »}

J.H. Bretz [1942] donne une classification des formes de creusement souterraines se référant à l'hypothèse de W.M. Davis [1930].

Il considère comme "phréatiques » les réseaux-éponges, les réseaux maillés, des salles horizontales creusées le long de strates fortement redressées, les anastomoses, les coupoles, des formes en «poche », les plafonds ou les parois déterminés par une diaclase (notamment les diaclases transversales), les tubes (dont il attribue cette interprétation à Clyde Malott), les arches rocheuses souterraines.

Et, pour les circulations « vadoses », elles détermineraient les méandres entaillant les parois, la rainure de niveau, le dome-pit et ses cannelures, les coups de gouge, les 
marmites de géants ; puis, en eaux stagnantes, le boxwork, les dissolutions affectant les joints stylolitiques et des blocs tombés.

Certains aspects de ce classement peuvent paraître contestables : il ne permet pas de comprendre l'existence d'anastomoses, de pendants dans le lit rocheux de cours d'eau aériens, comme l'Ardèche par exemple, ou des entrées de grottes (perte de Minerve, Hérault ; Mitchelstown Cave, Irlande). Pour les cannelures souterraines, elles semblent résulter de processus assez divers, et ne peuvent par elles-mêmes constituer une preuve d'écoulement libre.

Du reste, « les formes les plus remarquables des conduits [que l'on peut] supposer liés aux crues sont précisément celles considérées depuis des décennies comme indiquant une origine phréatique des grottes... La confusion avec un développement phréatique réel est compréhensible, puisque le régime de crue peut être considéré comme un retour aux conditions "phréatiques" dans la partie inférieure de la zone vadose » [A.N. Palmer 1972]. Déjà E.A. Glennie [1950] admet que les conduits « vadoses » peuvent acquérir la forme caractéristique de ceux qui sont «phréatiques ».

Les idées des auteurs américains ont été tardivement vulgarisées en France, par J. Corbel [1951], puis B Gèze [1965]. Elles ont d'ordinaire été réduites à une opposition entre les formes se rapprochant du tube ou résultant d'une dissolution différentielle, réputées «phréatiques », et les formes de surcreusement vertical, considérées comme «vadoses ». Or ces surcreusements se rencontrent aussi dans les conduits actifs suspendus, sans relation prouvée avec une descente du niveau de base.

Dans l'intervalle, P. Chevalier [1944] avait distingué deux actions essentiellement mécaniques : d'abord le creusement sous pression, examiné ci-dessus, puis une «érosion normale, en écoulement libre », surtout tributaire de la gravité. Ces idées, proches de celles des auteurs américains suscitent les mêmes objections. Puisque la mise en solution est toujours liée au contact de l'eau et de la roche, aucune forme de dissolution ne peut être spécifique d'un fonctionnement en écoulement libre ou en régime noyé. Le changement de régime d'écoulement est donc inefficace en soi, et ne peut expliquer le «méandre » décrit par P. Chevalier, dit maintenant « en trou de serrure »; celui-ci résulte en fait, le plus souvent, du recul d'une cascade.

\section{Le «Four-state Model » de D.C. Ford et R.O. Ewers [1978]}

Selon la figure 14 , qui se présente comme une proposition de synthèse d'hypothèses précédentes, les profils en long des conduits doivent être en escalier, éventuellement en montagnes russes, lorsque les surfaces de discontinuité géologiques sont rares ; et presque rectilignes, donc directement sous la surface de la nappe, lorsqu'elles sont denses. La vocation de la figure 14 est donc d'exprimer que, moins les surfaces de dis- 
continuité sont denses, plus la circulation initiale devrait descendre en profondeur ; autrement dit, plus la perméabilité est faible, plus l'eau descend profondément !

Dans la pratique, on constate l'inverse : on trouve des profils en long en montagnes russes dans les karsts de montagne, donc quand les fractures sont denses [dans le Hölloch en Suisse - A. Bögli 1980, par exemple]. Et des profils en long à pente régulière ne sont pas nécessairement liés à l'abondance de fractures, ni à la surface de la nappe, même lorsqu'ils n'utilisent pas un joint de stratification.

Pour les auteurs, le schéma de la figure 14 aurait aussi une valeur évolutive, puisqu'ils parlent de «Four-state Model », faisant l'hypothèse que la fracturation et la dissolution lente des petites fissures augmentent au cours du temps.

Pour P. Chevalier [1944], on l'a vu ci-dessus, une circulation en régime noyé n'est pas tributaire de la gravité. À l'inverse D.C. Ford [1971] admet qu'elle suit fréquemment un joint de stratification selon la ligne de plus grande pente (figure 15). Pourquoi selon la ligne de plus grande pente?

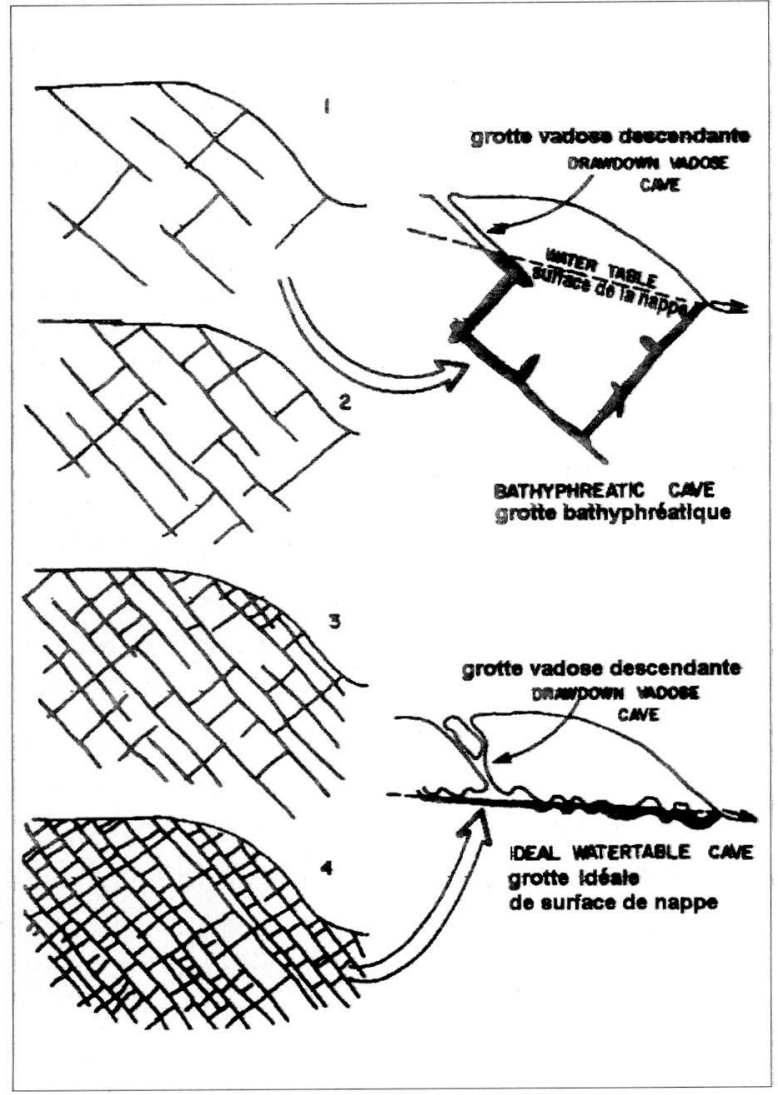

Figure 14 - En régime noyé, «Les quatre stades de fréquence de fissures sont représentés dans le cas de strates fortement pentées, et où les sorties du système sont dans l'aval-pendage. Les systèmes qui se sont développés dans les stades 1 et 4 sont représentés.» (d'après FORD, EWERS 1978). (The four state of fissure frequency drawn for the case of steeply deeping strata where the system outlets are in general direction of true type. Developed systems for states 1 and 4 are shown ) 


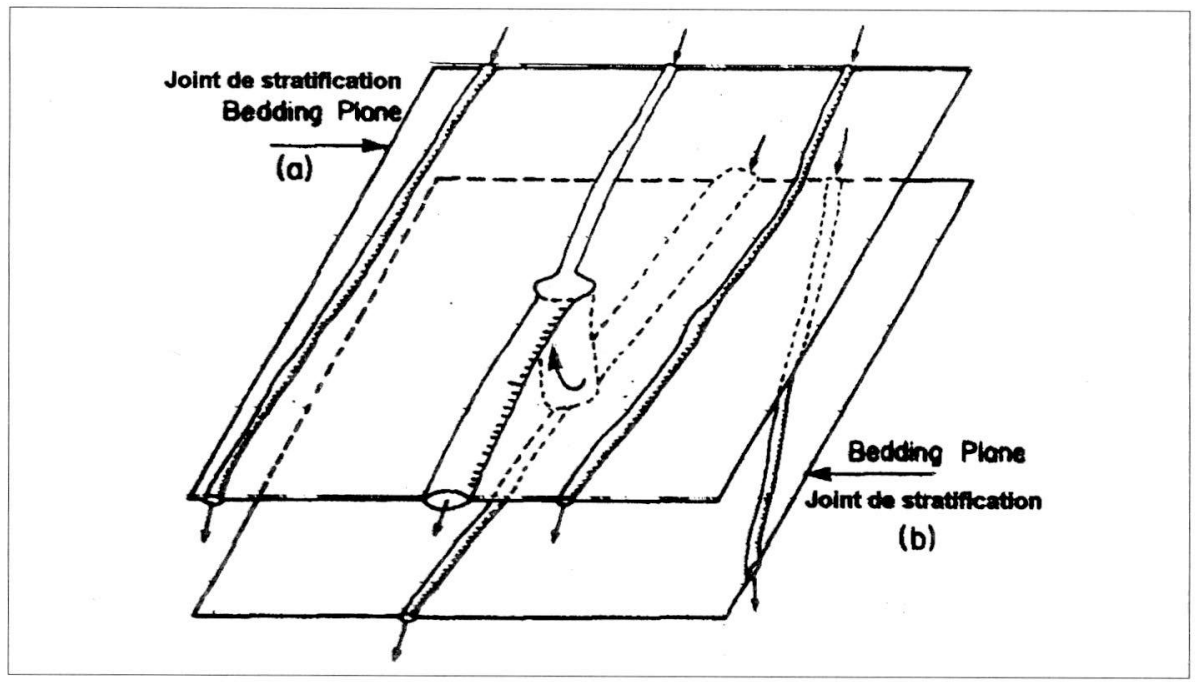

Fig. 15 - Des familles de "tubes de pendage" (de direction proche du pendage vrai) dans des joints de stratification, creusés successivement, sont les éléments souterrains de base, à partir desquels sont construits beaucoup de réseaux de dimensions explorables. Il sont souvent connectés par des "cheminées de diaclases" par lesquelles les eaux souterraines montent du joint de stratification inférieur (a) jusqu'au supérieur (b) (d'après FORD, EWERS 1978) (Families of "dip tubes" (oriented proximate to true dip) in successive penetrate bedding planes are the basic cave units from which many cave systems of explorable dimensions are built. They are connected commonly by "joint chimneys" up which groundwater flows from a lower plane (b) to a higher plane (a))

\section{L'hypothèse du creusement initial en un point favorable}

Des observations de terrain et des raisonnements théoriques ont conduit W. Maucci [1952] à supposer que la karstification peut débuter par le creusement d'un puits à l'intérieur du massif, en partant d'un point singulier lié à la structure géologique.

Sans qu'il y ait de relation, semble-t-il, la même hypothèse se retrouve ailleurs. La cavité correspondant dite : «fuseau »[W. Maucci 1952 - figure 16], «puits primaire » [Y. Quinif 1975], se serait creusée à partir d'un « foyer » [«foci» en anglais - D.J. Lowe 1992]. Chez ce dernier, le creusement débuterait au niveau d'un « inception horizon », défini comme «tout élément d'une séquence carbonatée, litho-stratigraphiquement contrôlée qui, en raison d'une différence physique, lithologique, chimique par rapport au faciès carbonaté prédominant dans la séquence, favorise passivement ou activement l'inception [= le début] de l'activité de dissolution ». Comme l'auteur le dit, cette définition n'apporte aucun concept nouveau ; elle omet par contre l'influence des fractures et celle, plus rare, d'une porosité efficace de la roche. 


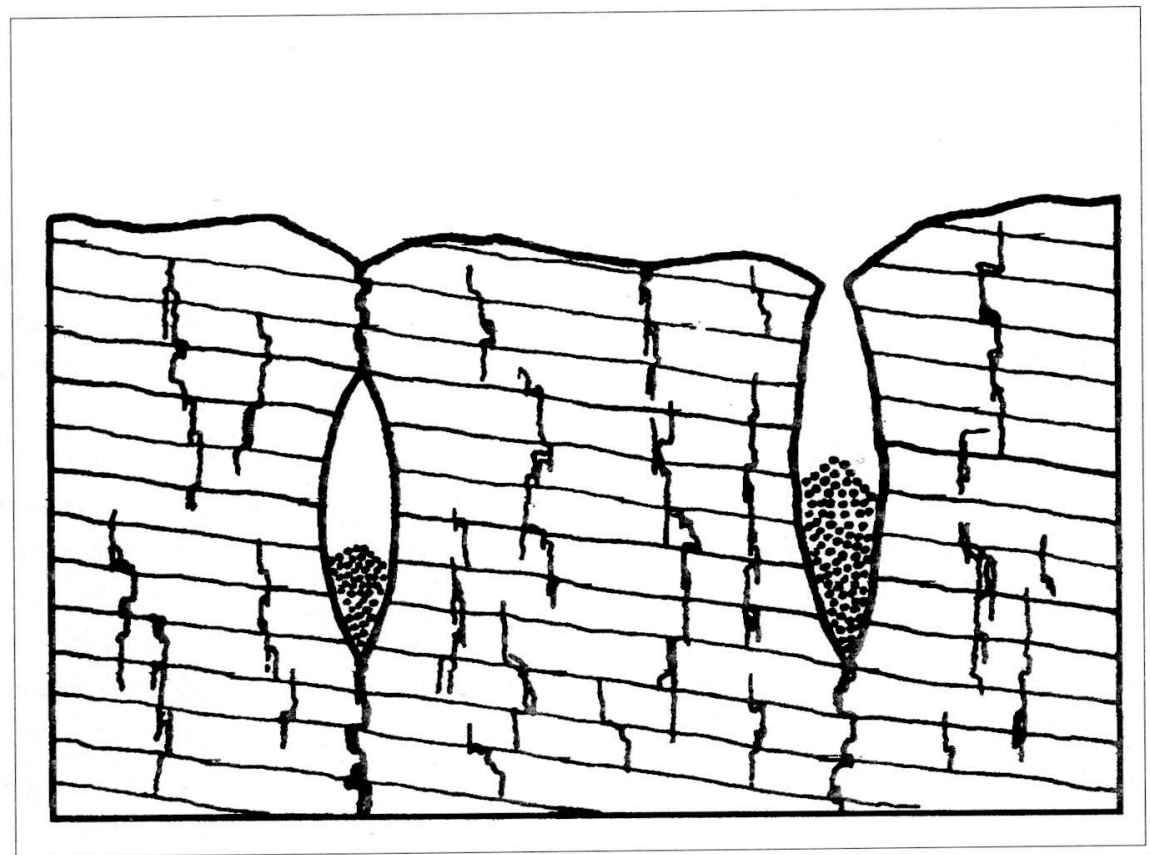

Fig. 16 - Section à gauche, d'un fuseau initial, et à droite, d'une « cavité jeune " par ouverture d'un fuseau à l'extérieur (d'après MAUCCI 1952) (On the left, cross section of an initial aven, and on the right, cross section of a «young cave» by opening of a aven)

Aucun de ces auteurs ne dit comment la cavité embryonnaire, par définition isolée dans un milieu presqu'imperméable, est alimentée puis drainée ; c'est-à-dire comment elles peut se creuser, puisqu'il n'y a de creusement que par la circulation. Des formes observées ailleurs, que l'on pourrait considérer comme comparables, ont été interprétées comme secondaires [C.A. Malott 1938 ; S. Jaillet 1995].

Dans des mines, ont été décrites des « cavités non intégrées » [non integrated caves - D.C. Ford et R.O. Ewers 1978], c'est-à-dire apparemment qui ne sont pas reliées à un réseau. Ces auteurs admettent qu'elles résultent de «processus diagénétiques, syngénétiques, etc. » ou de la « conjonction de ces processus avec la dissolution par des eaux météoriques », autrement dit qu'elles sont au plus secondairement karstique. Dans les conditions même de leur découverte lors de travaux miniers, il est pratiquement toujours impossible de démontrer que ces cavités sont indépendantes de toute autre système de vides karstiques. 


\section{L'évolution proprement dite}

\section{Variations du niveau de base}

L'explication proposée pour la quasi-totalité des évolutions de systèmes karstiques est basée sur une descente du niveau de base. Si des descentes du niveau de base sont effectivement responsables de très nombreux étagements de conduits, il est moins certain que chaque «stationnement» du niveau de base soit corrélable avec un niveau de circulations, puisque celles-ci demeurent souvent suspendues.

Mais les descentes du niveau de base général alternent avec des montées, de sorte que la distinction entre des circulations se poursuivant ou non sous le niveau marin, dites « holokarstiques » ou « mérokarstiques » [J. Cvijic' 1925], n'a plus de signification.

Comme les terrasses d'érosion des rivières sont des témoins de descentes du niveau de base, elles peuvent être corrélables avec des niveaux d'émergences. Pour les terrasses d'accumulation, qui sont des témoins d'ennoyage, une corrélation semblable [B. Gèze 1958] suppose qu'un processus d'érosion (le creusement d'un conduit) est tributaire d'un processus de sédimentation extérieur (le dépôt de la terrasse); tandis que des conduits existaient déjà à des niveaux inférieurs !

\section{Evolution de formes superficielles}

Selon une hypothèse, due pour une part à J. Cvijic' [1927], les dolines « représentent le premier effet de la karstification », l'eau s'évacuant par les fissures de la roche. Comment celles-ci, supposées non encore élargies, pourraientelles évacuer l'eau et les matériaux qui s'accumulent au fur et à mesure du creusement de la doline ? Les formes karstiques superficielles sont nécessairement secondaires par rapport à celles du karst profond.

J. Cvijic' [1927] suppose encore que les dolines s'élargissent progressivement et, par coalescence, donnent naissance à des dépressions karstiques plus volumineuses : ouvalas et poljés. Certes les poljés se sont établis dans des dépressions tectoniques, mais ils sont plus vastes « grâce à un large développement des ouvalas, grandes et petites ». Il n'explique pas en détail comment se produit cette coalescence, dont des exemples sont très rarement signalés. Et le phénomène est contesté par J. Roglic' [1939].

J. Cvijic' [1927] s'opposait à la notion de doline d'effondrement, considérant que toutes les dolines étaient de dissolution. À l'inverse, pour quelques auteurs [P. George 1948, P. Birot 1966], les canyons résultent presqu'automatiquement de l'effondrement de conduits souterrains. 


\section{Les classifications des karsts}

Les nombreuses tentatives de classification des karsts ne sont pas, comme on pourrait s'y attendre, issues de synthèses successives permettant de rattacher des critères morphologiques aux catégories proposées. Si la notion de facteur est sousjacente à toutes ces classifications, les observations liant la karstification à ces facteurs sont rares.

Les réserves émises ci-dessus sur ce que l'on sait du rôle du climat conduisent à considérer avec prudence même ce que l'on croit savoir des formes superficielles. Notamment dans des karsts étagés en raison de leurs altitudes croissantes, les expressions de fluvio-karst, nivo-karst, glacio-karst, sont fréquemment employées.

Ces expressions sont ambiguës : par exemple, selon les auteurs, le glacio-karst est le fait que l'eau de fonte du glacier intervient à l'aval sur les processus karstiques ; ou bien l'action conjointe des phénomènes glaciaire et karstique ; ou encore une succession d'actions glaciaires et karstiques alternées, donc une évolution. Or, dans beaucoup de régions, les trois définitions peuvent s'appliquer. Pour qu'une région puisse être qualifiée de glacio-karst, il faudrait qu'une proportion majoritaire de formes souterraines et superficielles soient glacio-karstiques. La même difficulté existe pour le nivo-karst.

Le fluvio-karst est celui où des circulations superficielles demeurent actives malgré la karstification. Chez divers auteurs, ce sont les circulations suspendues parce qu'en position de karst barré, les bassins fermés, les poljés, les surfaces d'aplanissement actives ; les karsts polygonaux. Et le fluvio-karst s'étend bien entendu aux régions où des couches karstifiables s'intercalent avec d'autres qui ne le sont pas.

Que reste-t-il au karst ? Faut-il admettre que tout karst est un fluvio-karst, ou que l'expression fluvio-karst est creuse?

La distinction entre karst de montagne et karst de plateau [N. Llopis Llado 1950] n'est pas toujours aisée : certains karsts de plateau sont d'altitude supérieure à d'autres qualifiés de montagnards. Et les classifications en fonction du relief oublient d'ordinaire le karst de plaine [D. Balazs 1977].

Des subdivisions ont été proposées selon le nombre de phases intervenant dans la mise en solution des diverses roches [A.A. Cigna 1978]. Des indications concernant le gypse [P. Forti et E. Rabbi 1981] ou des roches entièrement siliceuses de la Montagne de Table (Pologne) conduisent à penser que des actions biologiques ou des processus chimiques mal connus peuvent intervenir dans la mise en solution de roches assez diverses. 


\section{Conclusion}

Beaucoup de reconstitutions du creusement karstique ont été faites par référence à des hypothèses dont le présent article note qu'elles sont hasardeuses. Le risque est alors d'attribuer à un type de fonctionnement ce qui résulte peut-être d'un autre. Il serait facile de multiplier les exemples; je m'en tiens à deux, résultant de l'hypothèse d'un creusement successif en régime noyé, puis en écoulement libre [W.M. Davis 1930 ; P. Chevalier 1944] :

Cette hypothèse conduit presqu'automatiquement à considérer comme datant d'un passé hypothétique un conduit fonctionnant en écoulement libre, sans se poser la question de savoir si sa genèse ne serait pas possible dans les conditions actuelles. Et, puisqu'il est maintenant dénoyé, on va rechercher quelle fut la descente du niveau de base permettant de l'expliquer.

- Presque tout ce qui résulte d'ennoyages fut compris comme résultant d'un fonctionnement «phréatique » initial ; ce qu'exprime la célèbre phrase «Tous les karsts sont des Florides soulevées » [P. Birot 1954], alors que la Floride est vraisemblablement un karst ennoyé, G. Chabot [1927] le notait déjà.

Certaines des hypothèses examinées dans cette note occupent une place importante dans la vision du creusement karstique la plus généralement admise ; et l'on comprend qu'elles semblent dès lors très difficiles à abandonner.

Si l'on écarte ces hypothèses, il faut reconstruire, c'est-à-dire mettre en place un autre ensemble d'hypothèses. Ce à quoi je m'emploie. Puis d'autres abattront ces murs ${ }^{*}$ que je tente d'édifier.

\section{Remerciements}

Merci à Alfredo BINI, Yann CALLOT, Jacques CHABERT, Jean-Jacques DELANNOY, Guilhem FABRE, Christophe GAUCHON, Baudoin LISMONDE, Richard MAIRE, Philippe RENAULT $(\dagger)$, Jean-Noël SALOMON pour leur cordiale contribution à cette réflexion (même lorsqu'elle heurtait certaines de leurs convictions).

\footnotetext{
* “La science progresse non seulement en construisant, mais en détruisant, en abattant des murs qui, au lieu d'être des piliers, ne lui sont plus que des obstacles “ [EÖTVÖS]. Les matériaux de démolition sont souvent ceux des constructions nouvelles. La considération, voire l'amitié que j'ai pour ceux qui ont construit les murs que je prétend s' ébranler ne sont pas altérées de ce fait.
} 


\section{Bibliographie}

ANONYME, 1964 - Inédit.

AUBERT D., 1975 -L'évolution du relief jurassien. Eclogae Geol. Helvetiae 68 (1): 1-64.

AUDRA P., 1994 - Karsts alpins. Genèse de grands réseaux souterrains... - Karstologia Mémoires 5: 1-279.

BAKALOWICZ M., 1979 - Contribution à la géochimie des eaux, à la connaissance de l'aquifère karstique et de la karstification. Thèse de Doctorat d'Etat Univ. Paris 6: 1- 269.

BALAZS D., 1977 - The geographical distribution of karst areas. Proc. 7th. Intern. Speleological Congress, Sheffield: 13-15.

BARTHOLEYNS J.P., 1987 - Réseau Siebenhengste-Hogant. La crue du siècle et - 1000 $m$ à la clé. Spelunca 5: 27-29.

BIROT P., 1954 - Problèmes de morphologie karstique. Annales de Géographie LXIII/337: 161-192.

BIROT P., 1966 - Le relief calcaire. C.D.U., Paris: 1- 238.

BOEGAN E., 1938 - Il Timavo, studio sull' idrografia carsica subaerea e sotteranea. Mem. Ist. It. Speleologia, Serie Geologica e Geofisica II: 1- 251.

BÖGLI A., 1964 - Un exemple de complexe glacio-karstique, le Schichttreppenkarst ; "Karst et climats froids". Rev. Belge Géographie 88 (1-2): 63-82.

BÖGLI A., 1980 - Karst hydrology and physical speleology (trad. de Karsthydrographie und physische Speläologie, par J.C. SCHMID, Springer Verlag, Berlin: 1270.

BONTE A., 1958 - Réflexions sur l'origine des bauxites et sur l'altération superficielle des calcaires. Congr. Soc. Sav. Aix- Marseille, Section Sciences: 147-165.

BRETZ J H., 1942 - Vadose and phreatic features of limestone caverns J. Geology 50, 6/II: 675-811.

BRETZ J H., 1956 - Caves of Missouri. State of Missouri, Geological survey and water resources, Missouri (2) XXXIX, I-XXII + 1-490.

BROOK G.A., FORD D.C., 1976 - The Nahanni North karst: a questionmark on the valid- 
ity of the morphoclimatic concept of karst development. Actes $6^{\circ}$ Congr. Int. Spéléologie, Olomouc, 2: 43-57.

CALLOT Y., 1979 - A propos des plateaux ardéchois: Karst, Rapports fond-surface et évolution des paysages calcaires ou en roche perméable cohérente. Essai sur les paramètres influant dans la formation des paysages calcaires ou en roche perméable cohérente. Thèse $3^{\circ}$ Cycle Reims Géogr. Physique: 1 384.

CHABOT G., 1927 - Les plateaux du Jura central (thèse). Public. Fac. Lettres Univ. Strasbourg, Fasc. 41, Les Belles Lettres, Paris: 1- 350.

CHARTIER M.M., 1971 - Sur les cavités souterraines de la craie du massif de l'Othe. Actes Colloque Int. Karstologie et Spéléologie Languedoc-Périgord, Commission des Phénomènes Karstiques du Comité National de Géographie: 105-115.

CHEVALIER P., 1944 - Distinctions morphologiques entre deux types d'érosion souterraine. Rev. Géogr. Alpine XXXII/III: 475-486.

CHOPPY J., 1997 - Les deux faces de la pensée de Martel. Int. J. Spel., Phys. Spel. 26(3/4): 3-19.

CIGNA A.A., 1978 - A classification of karstic phenomena. Int. J. Spel. 10(1): 3-9.

CIGNA A.A., 1983 - Alcune considerazioni preliminari sull'erosione per cavitazione. Le grotte d'Italia, s. 4, XI:479-486.

CIRY R., 1959 - Une catégorie spéciale de cavités souterraines: les grottes cutanées. Ann. Spéléol., 14: 23-30.

CORBEL J., 1951 - Les études sur le karst depuis dix ans. Rev. Géogr. Lyon XXVI/1: 6770.

CORBEL J., 1957 - Les karsts du Nord-Ouest de l'Europe et de quelques régions de comparaison, (thèse). Rev. Géogr. Lyon, Mém. et Docum. Inst. Et. Rhodaniennes 12: 1-541.

CORBEL J., 1958 - Karsts du Yucatan et de la Floride. Bull. Assoc. Géogr. Fr. n 282-283: 2-14.

CORBEL J., 1963 - Marmites de géants et microformes karstiques. Norois, Poitiers, 10/38: 121-132. 
COWARD J., 1971 - Direct measure of erosion in a streambed of a West Virginia cave. Caves and Karst: 13(5): 39.

CRAMER H., HELLER F., 1933/1935 - Das Karstphänomen im Grundgips des fränkischen Keupers. Sonderdruck aus den Mittheilungen über Höhlen- und Karstforschung, 1933/4: 21-28; 1934: 1-7, 65-73, 97-107; 1935: 92-99.

CURL R.L., 1966 - Scallops and flutes. Trans. Cave Research Group G.B. 7: 121-162.

CVIJIC' J., 1909 - Bildung und Dislozierung der dinarischen Rumpfflächen. Petermanns Geogr. Mittheil. 6: 121-157, 177; 7: 153-163; 8:177-181.

CVIJIC' J., 1925 - Types morphologiques des terrains calcaires. C.R. Acad. des Sc. Paris 180, 23 Févr: 592-594, 9 Mars: 757-758 ; 30 Mars:1038-1040.

CVIJIC' J., 1927 - La géographie des terrains calcaires. Monogr. Acad. Serbe Sc. Arts CCCXLI, Classe des Sc. Mathématiques et Naturelles n² 26, Beograd 1960: 1-212.

DAVIES W.E., 1951 - Mechanics of cavern breakdown. N.S.S. Bull. 13: 36- 43.

DAVIS W.M., 1930 - Origin of limestone caverns. Bull. Geol. Soc. America, 41: 475-628.

DELANNOY J.J., 1997 - Recherches géomorphologiques sur les massifs karstiques du Vercors et de la transversale de Ronda (Andalousie). Thèse d'état en Géographie, Grenoble, Presses Universitaires du Septentrion, Villeneuve d'Ascq, 1998,: 1-678.

DEMANGEOT J., 1965 - Géomorphologie des Abruzzes adriatiques. Mém. et Doc. CNRS h.s.: 1- 403.

DREYBRODT W., 1987 - The kinetics of calcite dissolution and its consequences to karst evolution grom the initial to the mature state. The NSS Bulletin 49(2): 3149.

EK C., 1970 - Carte géologique de la grotte de Remouchamps - Les influences structurales sur la morphologie de la grotte de Remouchamps (Belgique). Ann. Soc. Géol. Belgique 93/II: 287-304.

ERASO A., 1986 - Metodo de prediccion de las direcciones principales de drenaje en el karst. Kobie, Bilbao, 15/1985-86: 15-165.

ERASO A., NAVARRO J.V., PARRA F., SAINT-AUBIN J., VALDES C., CANO J.P., 
RIBELLES J., DEL VAL J. 1 BERMEJO F., 1983, Problemas de fugas a traves del karst en la presa de Tous (España) ; Intern. Journal of Speleology $13,73-154$

FORD D.C., 1971 - Geological structure and a new explanation of limestone cavern genesis. Trans. Cave Research Group G. B., 13(2): 81-94.

FORD D.C., EWERS R.O., 1978 - The development of limestone cave systems in the dimensions of lenght and depth. Int. J. Spel., 10(3-4): 213-244.

FORTI P., RABBI E., 1981 - The role of $\mathrm{CO}_{2}$ in gypsum speleogenesis : $1^{\circ}$ contribution. Int. J. Spel., $11: 207-218$.

FOURNEAUX J.C., 1994 - Analyse des conditions de développement de la karstification profonde. Karstologia 23: 19-22.

GEORGE P., 1948 - Quelques formes karstiques de la Croatie occidentale et de la Slovénie méridionale - Yougoslavie. Ann. Géogr. 308: 298-307.

GÈZE B., 1958 - Sur quelques caractères fondamentaux des circulations karstiques. Ann. Spéléol. XIII: 5-22.

GÈZE B., 1965 - Les conditions hydrogéologiques des roches calcaires. Chronique d'Hydrogéologie B.R.G.M. 7: 9-39.

GÈZE B., 1973 - Les phénomènes karstiques. In: GOGUEL J., Géologie II - L'évolution de la terre ; "Encyclopédie de la Pléiade" ; Gallimard éd.: 70-102.

GLENNIE E.A., 1950 - Further notes on Ogof Ffynnon Ddu. Trans. Cave Research Group 1(3): 1-47.

GROVES C.G., HOWARD A.D., 1994 - Early development of karst systems. 1. Preferential flow path enlargement under laminar flow. Water Resources Research 30/10: 2837-2846.

GRUND A., 1903 - Die Karsthydrographie, Studien an Westbosnien. Penck's Geogr. Abhandl., Leipzig, VII(3): 103-200.

GRUND A., 1914 - Das geographische Zyklus in Karst. Zeitschrift Gesellsch. für Erdkunde, Berlin, 8: 621-640.

GUERIN R., 1973 - Un exemple du rôle de la tectonique et de la microtectonique dans la géométrie des écoulements karstiques fossiles et actuels : le Bas 
Vivarais calcaire. Thèse $3^{\circ}$ Cycle Tectono-Physique, Montpellier, 1-110., 1-132.

HERAK M., STRINGFIELD V.T., 1972 - Important karst regions of the northern hemisphere. Elsevier, Amsterdam, I-XIV+1-551.

HERNANDEZ, 1984 - Du nouveaux au Trou du vent de Caousos-Fontesborbes livre un peu son secret. Speleoc 27: 6 .

HJULSTRÖM, 1935 - Studies of the morphological activity of rivers as illustrated by the river Fyris. B. Geol. Inst. Uppsala: 221-527.

HORN G., 1947 - Karstuler $i$ Nordland. Norges Geologiske Undersökelse nr. 165: 1-77.

JAILlET S., 1995 - Le géosystème karstique du Rupt du Puits: Étude morphologique de l'exo- et de l'endo-karst.Mém. de Maitrise, Metz, Géographie: 1-173.

JEANNIN P.Y., 1989 - Paramètres physico-chimiques des eaux dans le karst. Diplôme $3^{\circ}$ cycle en Hydrogéologie, Neuchâtel, 1: 1-55; Remplissages karstiques du réseau des Sieben Hengste . 2: 1-61.

JEANNIN P.Y., 1996 - Structure et comportement hydraulique des aquères karstiques. Thèse de doctorat, Université de Neuchâtel: 1- 237.

JENNINGS J.N., LOWRY D.C., 1974 - The karsts of Nullarbor (Australia). Zeitschr. f. Geomorphologie NF, Berlin-Stuttgart, 18(1): 35-81.

JULIAN M., 1992 - Quelques réflexions théoriques sur le karst. In: "Hommage à Jean Nicod - Karst et évolutions climatiques", Presses universitaires de Bordeaux: 31-42.

KING F.H., 1899 - Principles and conditions of the movement of ground water. Nineteenth Annual Report United States Geological Survey, Part II: 58-384.

KIRALY L., 1968 - Eléments structuraux et alignement de phénomènes karstiques (région du gouffre du Petit-Pré de Saint-Livres, Jura Vaudois). Bull. Soc. Neuchâtel. Sc. Nat. 91: 127-146.

LAURITZEN S.E., 1989 - Shear, tension or both. A critical view on the prediction potential for caves. Actes $10^{\circ}$ Congr. Int. Spél., Budapest, I:118-120.

LEHMANN H., 1953 - Karst-Entwicklung in den Tropen. Die Umschau in Wissenschaft und Technik Jg. 53, Frankfurt a. Mein, 18: 559-562. 
LEHMANN H., 1960 - La terminologie classique du karst sous l'aspect critique de la morphologie climatique moderne. Rev. Géogr. Lyon $\mathbf{X X X V ( 1 ) : ~} 6$ p., 8 pl.

LEHMANN O., 1932 - Die Hydrographie des Karstes. In: "Enzyklopädie der Erdkunde", F. Deuticke, Leipzig und Wien, 212 p. (analyse de MARTONNE 1933)

LISMONDE Baudoin, 2000 - Inédit.

LLOPIS LLADO N., 1950 - Sobre algunos principios fundamentales de morfologia y hidrologia carstica. Estudios geograficos n XI(41: 643-679 ; aussi: $1952-$ Speleon III: 33-69.

LOWE D.J., 1992 - The origin of limestone caverns. An inception horizon hypothesis. Thèse, Manchester Polytechnic: I- XX+1-512.

MAIRE R., POMEL S., 1995 - Formation d'un karst conique à partir de la couverture de flysch. «Donghe 92. Karsts de Chine centrale », Karstologia Mém. 6: 130141.

MALOTT C.A., 1938 - Invasion theory of cavern development. Geol. Soc. America Proc. 1936: 323.

MANGIN A., 1969 - Etude hydraulique du mécanisme d'intermittence de Fontestorbes (Belesta, Ariège). Ann. Spél. 24(2): 253-258; 260-261; 268- 274.

MANGIN A., 1974/1975 - Contribution à l'étude hydrodynamique des aquifères karstiques. Ann. Spél. 29(3): 282-332; 29(4): 495-601; 30(1): 21-124.

MANGIN A., 1982 - L'approche systémique du karst, conséquences conceptuelles et méthodologiques. Reunion Monografica sobre el karst, Larra:141-57.

MANGIN Alain, 1995 - Inédit.

MANGIN A., BAKALOWICZ M., 1990 - Le karst conique : sa genèse à partir de l'exemple du karst du sud de la Chine. C.R. Acad.Sciences, Paris, 310, série II: 301-307.

MARTEL E.A., 1921 - Nouveau Traité des Eaux Souterraines. Doin, Paris: 1-838; reprint Jeanne Laffitte 1983.

MARTEL E.A., 1930 - La France Ignorée II (Des Ardennes aux Pyrénées). Delagrave, Paris: $1-306$ 
MARTIN P., 1991 - Hydromorphologie des géosystèmes karstiques des versants Nord et Ouest de la Sainte Baume. Thèse Géographie Aix-Marseille: 1- 326.

MARTINI J., 1960, Note sur l'érosion inversée ; Stalactite IV/5, 125-32

MARTONNE E. de, 1926 - Traité de géographie physique. A. Colin $4^{\circ}$ éd. Paris, 2 (Le relief du sol): 499-1057.

MAUCCI W., 1952 - L'ipotesi del "erosione inversa" come contributo allo studio della speleogenesi. Boll. Soc. Adriatica di Sc. Nat., Trieste 1951-1952, XLVI, 160. (réédité avec une discussion dans Le Grotte d'Italia (4a) IV, 1973: 235285, paru en 1975).

MÉGNIEN C., 1964 - Observations hydrogéologiques sur le Sud-Est du Bassin de Paris Les circulations aquifères dans le Jurassique et le Crétacé de l'Yonne. Mém. B.R.G.M. 25: 1-287.

MYLROIE J.E., CAREW J.L., 1987 - Field evidence of the minimum time for speleogenesis. The NSS Bulletin, 49(2): 67-72.

NICOD J., 1967 - Recherches morphologiques en Basse Provence calcaire. Thèse Doctorat d'Etat, Publ. Université d'Aix-en-Provence; Ophrys, Gap: 1-560.

NICOD J., 1982 - Niveaux de base régressifs et formes karstiques submergées; "Karsts littoraux", Mém. A.F.K. 4: 67-74.

PALMER A.N., 1972 - Dynamics of a Sinking stream system: Onesquethaw cave, New York. Bull. NSS 34(43): 89-110.

PALMER A.N., 1981 - Hydrochemicals factors in the origin of limestone caves. Proc. 8th Int. Congr. Spel., Bowling Green, 1: 120-122.

PFEFFER K.H., 1995 - Karstresearch - a traditional sience involving recent applied tasks. " Environmental effects on karst terrains », Acta geografica, Szeged (Hongrie) XXXIV: 7-24.

PUIG J.M., 1990 - Le système karstique de la fontaine de Vaucluse. (Thèse), Document BRGM 180: 1-208.

PUYOO S., 1976 - Etude hydrogéologique du massif karstique d'Arbas (Haute Garonne).

Thèse $3^{\circ}$ Cycle Univ. Pierre et Marie Curie, Paris: 1-193+8 p.

QUINIF Y., 1975 - Etude spéléogénétique des avens-chantoirs belges. Speleologia Belgica 